\title{
Further explorations of Skyrme-Hartree-Fock-Bogoliubov mass formulas. XII: Stiffness and stability of neutron-star matter
}

\author{
S. Goriely, ${ }^{1}$ N. Chamel, ${ }^{1}$ and J. M. Pearson ${ }^{2}$ \\ ${ }^{1}$ Institut d'Astronomie et d'Astrophysique, CP-226, \\ Université Libre de Bruxelles, B-1050 Brussels, Belgium \\ ${ }^{2}$ Département de Physique, Université de Montréal, \\ Montréal (Québec), H3C 3J7 Canada
}

(Dated: October 27, 2018)

\begin{abstract}
We construct three new Hartree-Fock-Bogoliubov (HFB) mass models, labeled HFB-19, HFB20, and HFB-21, with unconventional Skyrme forces containing $t_{4}$ and $t_{5}$ terms, i.e., densitydependent generalizations of the usual $t_{1}$ and $t_{2}$ terms, respectively. The new forces underlying these models are fitted respectively to three different realistic equations of state of neutron matter for which the density dependence of the symmetry energy ranges from the very soft to the very stiff, reflecting thereby our present lack of complete knowledge of the high-density behavior of nuclear matter. All unphysical instabilities of nuclear matter, including the transition to a polarized state in neutron-star matter, are eliminated with the new forces. At the same time the new models fit essentially all the available mass data with rms deviations of $0.58 \mathrm{MeV}$ and give the same high quality fits to measured charge radii that we obtained in earlier models with conventional Skyrme forces. Being constrained by neutron matter, these new mass models, which all give similar extrapolations out to the neutron drip line, are highly appropriate for studies of the $r$-process and the outer crust of neutron stars. Moreover, the underlying forces, labeled BSk19, BSk20 and BSk21, respectively, are well adapted to the study of the inner crust and core of neutron stars. The new family of Skyrme forces thus opens the way to a unified description of all regions of neutron stars.

PACS numbers: 21.10.Dr, 21.30.-x, 21.60.Jz, 26.60.Dd, 26.60.Kp
\end{abstract}




\section{INTRODUCTION}

With a view to their astrophysical application in neutron-rich environments, we have for some years been developing a series of nuclear-mass models based on the Hartree-FockBogoliubov (HFB) method with Skyrme and contact-pairing forces, together with phenomenological Wigner terms and correction terms for the spurious collective energy; all the model parameters have been fitted to essentially all the experimental mass data. The different Skyrme forces used in each of our models up to and including HFB-17 [1] had the conventional form

$$
\begin{aligned}
v_{i j}= & t_{0}\left(1+x_{0} P_{\sigma}\right) \delta\left(\boldsymbol{r}_{i j}\right)+\frac{1}{2} t_{1}\left(1+x_{1} P_{\sigma}\right) \frac{1}{\hbar^{2}}\left[p_{i j}^{2} \delta\left(\boldsymbol{r}_{i j}\right)+\delta\left(\boldsymbol{r}_{i j}\right) p_{i j}^{2}\right] \\
& +t_{2}\left(1+x_{2} P_{\sigma}\right) \frac{1}{\hbar^{2}} \boldsymbol{p}_{i j} . \delta\left(\boldsymbol{r}_{i j}\right) \boldsymbol{p}_{i j}+\frac{1}{6} t_{3}\left(1+x_{3} P_{\sigma}\right) \rho(\boldsymbol{r})^{\alpha} \delta\left(\boldsymbol{r}_{i j}\right) \\
& +\frac{\mathrm{i}}{\hbar^{2}} W_{0}\left(\boldsymbol{\sigma}_{\boldsymbol{i}}+\boldsymbol{\sigma}_{\boldsymbol{j}}\right) \cdot \boldsymbol{p}_{i j} \times \delta\left(\boldsymbol{r}_{i j}\right) \boldsymbol{p}_{i j},
\end{aligned}
$$

where $\boldsymbol{r}_{i j}=\boldsymbol{r}_{i}-\boldsymbol{r}_{j}, \boldsymbol{r}=\left(\boldsymbol{r}_{i}+\boldsymbol{r}_{j}\right) / 2, \boldsymbol{p}_{i j}=-\mathrm{i} \hbar\left(\boldsymbol{\nabla}_{i}-\nabla_{j}\right) / 2$ is the relative momentum, $P_{\sigma}$ is the two-body spin-exchange operator, and $\rho(\boldsymbol{r})=\rho_{n}(\boldsymbol{r})+\rho_{p}(\boldsymbol{r})$ is the total local density, $\rho_{n}(\boldsymbol{r})$ and $\rho_{p}(\boldsymbol{r})$ being the neutron and proton densities, respectively. With this model we were able to fit with an rms deviation of $0.581 \mathrm{MeV}$ the 2149 measured masses of nuclei with $N$ and $Z \geq 8$ given in the 2003 Atomic Mass Evaluation [2] (AME), while at the same time constraining the underlying Skyrme force to fit properties of homogeneous neutron matter (NeuM), as determined by many-body calculations with realistic two- and three-nucleon

forces; the pairing force was fitted to the ${ }^{1} S_{0}$ pairing gaps of homogeneous nuclear matter of the appropriate charge asymmetry.

The constraint of the Skyrme force to NeuM enhances the reliability with which model HFB-17 can make mass predictions for the experimentally inaccessible highly neutron-rich nuclei that appear in the outer crust of neutron stars and that are involved in the $r$-process of stellar nucleosynthesis. Moreover, such a mass model can be used to extrapolate beyond the drip line to the inner crust of neutron stars, using the underlying force (BSk17 in the case of the HFB-17 mass model) to calculate the equation of state (EOS) in this region, i.e., the energy per nucleon as a function of density for a given temperature. Our confidence in this extrapolation derives not only from the fit of the interactions to NeuM but also from the precision fit to masses, which means that the presence of protons and the existence of inhomogeneities in the inner crust are well represented. Being able to use the same effective 
force for the outer and inner crusts means that a coherent treatment of the interface between the two regions becomes possible [3].

The fit to the calculated properties of NeuM suggests that the extrapolation beyond the drip line can be extended still further, into the homogeneous core of neutron stars, making it possible to use the model force to calculate the EOS of the matter of that region, the so-called neutron-star matter $\left(\mathrm{N}^{*} \mathrm{M}\right)$, which is highly neutron rich but contains also proton-electron pairs and possibly muons (and other particles, such as hyperons, quarks, etc., toward the center of the star). Being able to use the effective force in this region would provide a valuable tool for extending the information given directly by the realistic calculations of NeuM to which the force had been fitted. In particular it could be indispensable for a calculation of the EOS of $\mathrm{N}^{*} \mathrm{M}$, realistic calculations being extremely limited in this respect, if not non-existent. In this way we would open up the prospect of a unified treatment of all regions of the neutron star using a unique effective force; in particular a coherent treatment of the interface between the inner crust and the core would become possible, as for the interface between the inner and outer crusts.

However, in the case of HFB-17 and all our previous models, this development was frustrated by the fact that they predict NeuM and $\mathrm{N}^{*} \mathrm{M}$ to have polarized ground states that would lead to neutron stars being unstable against collapse to indefinitely high densities: the unpolarized state of NeuM to which our forces were fitted turns out not to be the ground state. We dealt with this problem in our latest published model, HFB-18 [4], by adding to the conventional form of Skyrme force (1) two extra terms, writing our complete Skyrme force as

$$
\begin{aligned}
v_{i, j}^{\prime} & =v_{i, j}+\frac{1}{2} t_{4}\left(1+x_{4} P_{\sigma}\right) \frac{1}{\hbar^{2}}\left\{p_{i j}^{2} \rho(\boldsymbol{r})^{\beta} \delta\left(\boldsymbol{r}_{i j}\right)+\delta\left(\boldsymbol{r}_{i j}\right) \rho(\boldsymbol{r})^{\beta} p_{i j}^{2}\right\} \\
& +t_{5}\left(1+x_{5} P_{\sigma}\right) \frac{1}{\hbar^{2}} \boldsymbol{p}_{i j} \cdot \rho(\boldsymbol{r})^{\gamma} \delta\left(\boldsymbol{r}_{i j}\right) \boldsymbol{p}_{i j}
\end{aligned}
$$

where the $t_{4}$ and $t_{5}$ terms are density-dependent generalizations of the $t_{1}$ and $t_{2}$ terms, respectively. The full formalism for this generalized Skyrme force is presented in the Appendix of Ref. [4]. With the new terms it was possible in the HFB-18 model to stop the unphysical transition of NeuM (and $\mathrm{N}^{*} \mathrm{M}$ ) to a spin-ordered state, with only an insignificant deterioration in the quality of the mass fit. Furthermore, in the HFB-18 model our adjustment of these new terms was minimal, in the sense that the three parameters of the $t_{5}$ term were chosen in a rather arbitrary way, subject only to the condition of stopping the unwanted 
transition, while the three parameters of the $t_{4}$ term were fixed by the equations

$$
\begin{gathered}
\beta=\gamma \\
t_{4}=-\frac{1}{3} t_{5}\left(5+4 x_{5}\right) \\
x_{4}=-\frac{4+5 x_{5}}{5+4 x_{5}}
\end{gathered}
$$

which ensure that the $t_{4}$ term cancels exactly the $t_{5}$ term in unpolarized homogeneous nuclear matter of any degree of charge asymmetry at all densities. This latter condition is not strictly necessary, since it can be violated significantly without compromising the quality of the mass fit or the stability of NeuM and $\mathrm{N}^{*} \mathrm{M}$ against a transition to a polarized state; we imposed it just to simplify the parameter search in the mass fit. Thus the prescription that we adopted for determining both the $t_{4}$ and $t_{5}$ terms in model HFB-18 leaves us with considerable flexibility for realizing further improvements, and in this article we begin to exploit these possibilities.

The main defect of the HFB-18 model that we address in this article is its lack of flexibility in fitting the EOS of NeuM at zero temperature. In developing this model we had no trouble in constraining to the EOS of Friedman and Pandharipande [5] (FP), which is based on a variational calculation using the realistic Urbana $v_{14}$ nucleon-nucleon force with the threebody force TNI. However, it is by no means clear that the FP EOS for NeuM is the correct one, since some quite different ones that are at least equally plausible have been published. For example, the EOS of Akmal et al. [6] labeled "A18 + $\delta v+$ UIX*", which we refer to as APR, is considerably stiffer. This EOS, like FP, is based on a variational calculation but uses the realistic Argonne A18 two-body force and the semi-phenomenological UIX* three-body force (see Ref. [6] for details of these forces); there is also a so-called relativistic boost correction $\delta v$. Actually, while the latter EOS is more recent and more complete, there have been some recent experimental indications that it might be too stiff [7]. On the other hand, several realistic EOSs for NeuM that are still stiffer than APR have been published. One such striking case is based on the same A18 two-body force as is APR, but uses a much more realistic three-body force and is calculated with the Brueckner-Hartree-Fock (BHF) method. This is the EOS labeled "V18" in Ref. [8], which we will refer to as LS2 (a very similar EOS, based on the quantum Monte Carlo method, has been published in Ref. [9]). 
In this article we make no assumption as to which of these three EOSs, FP, APR or LS2, is closer to reality, but rather generate a family of three mass models with effective forces constrained respectively to these three realistic EOSs. In this way we may reasonably hope with our family of effective forces to cover the range of possibilities left open by the present limitations of our knowledge. However, we find that as long as the conditions (3a) - (3c) are maintained it is impossible to constrain to any EOS stiffer than that of $\mathrm{FP}$ while maintaining a high quality mass fit. We shall see here that by releasing these conditions and exploiting the degrees of freedom associated with the $t_{4}$ and $t_{5}$ terms we can stiffen the EOS of NeuM without sacrificing any of the achievements of the HFB-18 model.

Other problems that appear to be inevitable in mass models based on the conventional form of Skyrme forces include an incorrect distribution of the total potential energy among the four two-body spin-isospin channels in symmetric nuclear matter (SNM), and Landau parameters with values indicating various unphysical instabilities in homogeneous nuclear matter. The new terms in $t_{4}$ and $t_{5}$ allow us enough flexibility to handle both of these problems as well as that of the stiffness of the EOS of NeuM.

Accordingly, we present in this article three new mass models, HFB-19, HFB-20, and HFB-21. The first of these, HFB-19 has its underlying force, BSk19, constrained, like BSk18 (the force of model HFB-18), to the FP neutron-matter EOS, while BSk20 and BSk21 (the forces of models HFB-20 and HFB-21, respectively), are constrained to the APR and LS2 neutron-matter EOSs, respectively. All three satisfy the required stability properties of nuclear matter, and a qualitatively acceptable distribution of the potential energy among the spin-isospin channels. In Sec II we describe the new models and many of their properties, with discussions of their symmetry energy and Landau parameters being treated separately in Secs III and IV] respectively. The behavior of these models in $\mathrm{N}^{*} \mathrm{M}$ is dealt with in Sec $\nabla$. We summarize our conclusions and discuss possible future developments in Sec VI. Appendix A describes some microscopic calculations that support our more phenomenological collective corrections, while Appendix B gives the expressions for the Landau parameters of neutron matter with our generalized Skyrme forces. 


\section{THE HFB-19, HFB-20, AND HFB-21 MASS MODELS}

\section{A. Principal features of models}

Our new HFB calculations for finite nuclei are performed almost exactly as for the HFB18 model [4]. In particular, the treatment of pairing is highly realistic. As usual, we take a contact pairing force that acts only between nucleons of the same charge state $q$,

$$
v_{q}^{\text {pair }}\left(\boldsymbol{r}_{\boldsymbol{i}}, \boldsymbol{r}_{\boldsymbol{j}}\right)=v^{\pi q}\left[\rho_{n}(\boldsymbol{r}), \rho_{p}(\boldsymbol{r})\right] \delta\left(\boldsymbol{r}_{i j}\right),
$$

where the strength $v^{\pi q}\left[\rho_{n}, \rho_{p}\right]$ is a functional of both the neutron and proton densities. But instead of postulating a simple functional form for the density dependence, as is usually done, we construct the pairing force by solving the HFB equations in uniform asymmetric nuclear matter with the appropriate neutron and proton densities, requiring that the resulting gap reproduce exactly, as a function of density, the microscopic ${ }^{1} S_{0}$ pairing gap calculated with realistic forces [10]. We follow our usual practice in finite nuclei of allowing the proton pairing strength to differ from the neutron pairing strength, and for allowing each of these strengths to depend on whether there is an even or odd number of nucleons of the charge type in question. These extra degrees of freedom are taken into account by multiplying the value of $v^{\pi q}\left[\rho_{n}, \rho_{p}\right]$, as determined by the nuclear-matter calculations that we have just described, with renormalizing factors $f_{q}^{ \pm}$, where $f_{p}^{+}, f_{p}^{-}$and $f_{n}^{-}$are free, density-independent parameters to be included in the mass fit, and we set $f_{n}^{+}=1$. (For more details see Refs. [1, 11].)

To the HFB energy calculated for the Skyrme and pairing forces we add a Wigner correction,

$$
E_{W}=V_{W} \exp \left\{-\lambda\left(\frac{N-Z}{A}\right)^{2}\right\}+V_{W}^{\prime}|N-Z| \exp \left\{-\left(\frac{A}{A_{0}}\right)^{2}\right\},
$$

which contributes significantly only for light nuclei $\left(A<A_{0}\right)$ or nuclei with $N$ close to $Z$. Our treatment of this correction is purely phenomenological, although physical interpretations of each of the two terms can be made [11, 12].

A second correction that must be made is to subtract from the HFB energy an estimate for the spurious collective energy. As described in Ref. [11], the form we adopt here is

$$
E_{\text {coll }}=E_{\text {rot }}^{\text {crank }}\left\{b \tanh \left(c\left|\beta_{2}\right|\right)+d\left|\beta_{2}\right| \exp \left\{-l\left(\left|\beta_{2}\right|-\beta_{2}^{0}\right)^{2}\right\}\right\}
$$


in which $E_{\text {rot }}^{c r a n k}$ denotes the cranking-model value of the rotational correction and $\beta_{2}$ the quadrupole deformation, while all other parameters are fitted freely. While the first term here represents the rotational correction, phenomenologically modified from its cranking-model value, the second term takes account of the deformation dependence of the vibrational correction [since $E_{\text {coll }}$ as given by Eq. (6) vanishes for spherical nuclei we must suppose that the vibrational correction for such nuclei is absorbed into the fitted force parameters]. We have tested [13] the reliability of the rotational part of this expression against an exact calculation made by Bender et al. [14], while in Ref. [15] we discussed the validity of our vibrational correction in terms of the constraints provided by shape isomers and fission barriers. Further validation of our collective correction comes from the microscopic calculations described in Appendix A.

The final correction that we make is to drop Coulomb exchange. This is a device that we have successfully adopted in our most recent models, beginning with HFB-15 [16], and it can be interpreted as simulating neglected effects such as Coulomb correlations, chargesymmetry breaking of the nuclear forces, and vacuum polarization.

The only difference between the present HFB calculations and those for the HFB-18 model [4] is that we now drop all the terms in $J^{2}$ and $J_{q}^{2}$ from the Hamiltonian density (A3) of Ref. [4], as is done in most parametrizations of the Skyrme force. We were able to accommodate these terms in our HFB codes without any problem, and our initial motivation for dropping them here was simply to make the forces more compatible with our EOS code for the inner crust of neutron stars [3], where the inclusion of these terms would have led to considerable complexity. However, omitting the time-even $J^{2}$ and $J_{q}^{2}$ terms requires, on the grounds of gauge invariance [17], that we drop also the time-odd terms in $\left(\mathbf{s}_{\mathbf{n}}+\mathbf{s}_{\mathbf{p}}\right) \cdot\left(\mathbf{T}_{\mathbf{n}}+\mathbf{T}_{\mathbf{p}}\right)$ and $\left(\mathbf{s}_{\mathbf{n}}-\mathbf{s}_{\mathbf{p}}\right) \cdot\left(\mathbf{T}_{\mathbf{n}}-\mathbf{T}_{\mathbf{p}}\right)$. This is ensured in the notation of Ref. [18] by setting $C_{0}^{T}=C_{1}^{T}=0$. In dropping these terms from the mass fit, which arise jointly from the $t_{1}, t_{2}, t_{4}$ and $t_{5}$ terms, their effect is to some extent absorbed by all the Skyrme parameters, notably $W_{0}$ (compare the values of this parameter shown in Table \ for force BSk18 with those shown for BSk19 - BSk21). In this way the contribution of the omitted terms will be at least partially simulated in the EOS calculations. At the same time, it will be seen in the following that this approximation does not lead to any deterioration in the global fit to masses or radii, although there are implications for properties depending on the time-odd terms in the Hamiltonian. In the present article such properties include the EOS of polarized nuclear 
matter, the distribution of the potential energy among the different spin-isospin channels, and the Landau parameters. The time-odd terms also play a role in the exact treatment of the masses of odd nuclei, but not in the equal-filling approximation [19], which we adopt here, as in all our previous articles.

\section{B. The data fits}

The parameters of the three new models, i.e., of the Skyrme and pairing forces, and of the Wigner and collective corrections, are fitted to the same set of mass data as was the HFB-18 model, i.e., the 2149 measured masses of nuclei with $N$ and $Z \geq 8$ given in Ref. [2]. In making these fits we imposed the NeuM constraints discussed in Sec I, with the FP EOS [5] defining force BSk19, the APR EOS [6] defining BSk20, and the LS2 EOS [8] defining BSk21 (throughout this article we assume zero temperature). The fits were also subject to our usual requirement that the isoscalar effective mass $M_{s}^{*}$ take the realistic value of $0.8 M$ in SNM at the equilibrium density $\rho_{0}$ (see the discussion in Ref. [20]); note that the isovector effective mass $M_{v}^{*}$ has no role to play in SNM, as can be seen from Eq. (10)). We likewise imposed a value of $J=30 \mathrm{MeV}$ on the symmetry coefficient; this choice is certainly consistent with all the available evidence coming from both experiment and theory, although the situation is somewhat ambiguous (see especially Sec IIIB). Also we required that the incompressibility $K_{v}$ fall in the experimental range $240 \pm 10 \mathrm{MeV}$ [21]. The values of the Skyrme, pairing and Wigner parameters resulting from these three fits are shown in Table \ $\left(\varepsilon_{\Lambda}\right.$ is the pairing cutoff parameter [1, 11]). The parameters of the collective correction of Eq. ([6]) are shown in Table [I], (All the expressions corresponding to the generalized Skyrme force (2) required here are given in, or can be trivially derived from, the Appendix of Ref. [4].)

Figure1 shows that each of the three new forces of this article, BSk19-BSk21, reproduces very well its realistic "target" EOS of NeuM. In this figure we show also the results of the realistic calculation "UV14 plus TNI" of Wiringa et al. [22], labeled here as WFF. This EOS uses the same realistic forces as FP, and like FP is based on a variational calculation; it agrees very closely with the EOS of FP, but runs to higher densities, and so generally we shall henceforth take WFF rather than FP as the reference EOS for BSk19. We have checked for all of our forces that the ground state of NeuM is unpolarized over the entire density range shown in Figure 1, that is, there is no ferromagnetic instability. At the same 


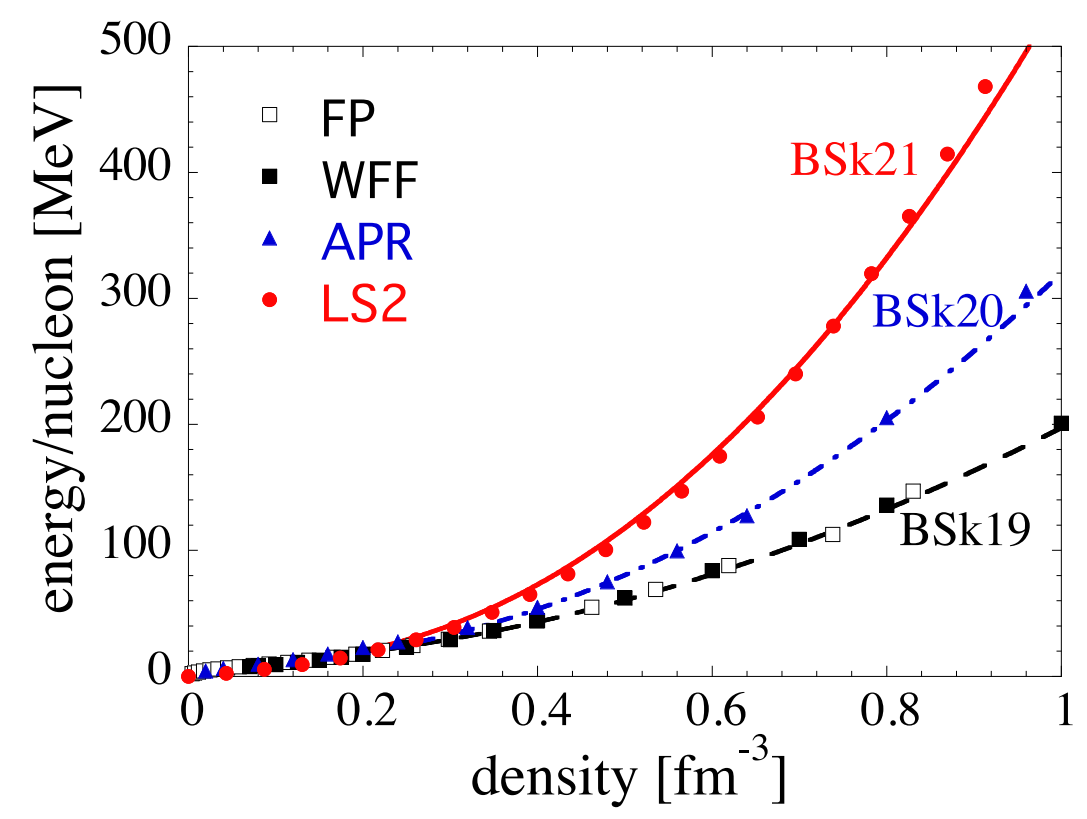

FIG. 1: (Color online) Zero-temperature EOSs for neutron matter (NeuM) with forces BSk19, BSk20 and BSk21. Also shown are the realistic EOSs FP [5], WFF [22], APR [6] and LS2 [8].

time, in fitting the force parameters we have also to check that the ground state of $\mathrm{N}^{*} \mathrm{M}$ is likewise stable against polarization, since the stability of NeuM does not guarantee that of $\mathrm{N}^{*} \mathrm{M}$ (see Sec $\mathrm{V}$ for further details of the $\mathrm{N}^{*} \mathrm{M}$ calculations). It is seen from Table $\square$ that we have relaxed the conditions (3a) - (3c) that we imposed on the search for model HFB-18 [4], which means that the $t_{4}$ and $t_{5}$ terms no longer exactly cancel in unpolarized homogeneous nuclear matter of arbitrary charge symmetry. This is the source of the extra flexibility that we have gained in the present work, although it makes the fitting process much more laborious.

Figure 2 is a low-density zoom of Figure 1, comparing our forces with the NeuM of FP and also that given by the BHF calculations of Baldo et al. [23] (the calculations of WFF are not taken to such low densities). This figure also shows the corresponding results for SNM. The excellent agreement of all our forces with the realistic calculations, which have served as the basis of a density functional for nuclear-structure calculations [23], will be seen.

The rms and mean (data - theory) values of the deviations between the measured masses and the predictions for the HFB-19, HFB-20 and HFB-21 models are given in the first and second lines, respectively, of Table III, where we also compare with HFB-18 [4]. With HFB21 we have achieved our best mass fit ever, although we do not regard its superiority over 


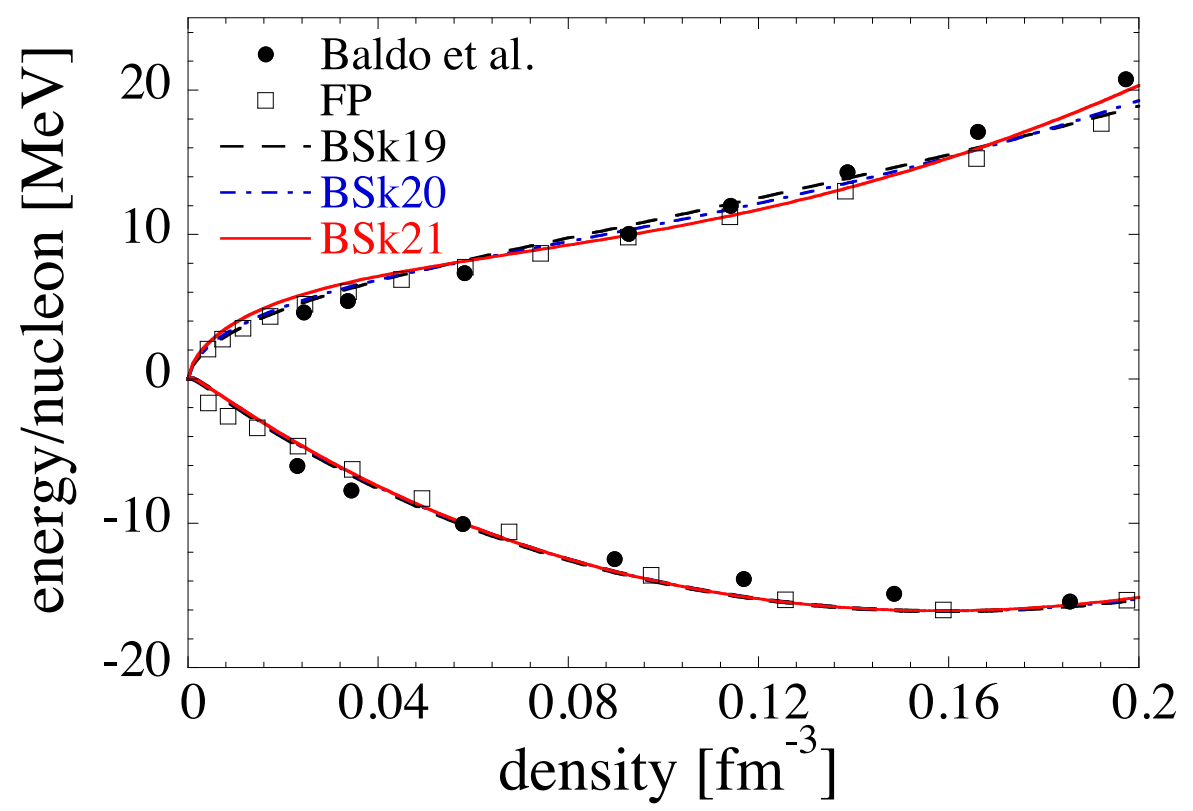

FIG. 2: Low-density EOS in NeuM and SNM of forces BSk19 - BSk21. We compare with the calculations of Refs. [5, 23].

the other three forces as significant. The next two lines of Table III show the deviations for the subset consisting of the most neutron-rich measured nuclei, here taken as those with a neutron separation energy $S_{n} \leq 5.0 \mathrm{MeV}$. All four models display a slight deterioration as we move into the neutron-rich region. (The rms deviation given by the finite-range droplet model (FRDM) [24] for this same subset is $0.910 \mathrm{MeV}$.) Lines 5 - 8 show the deviations for the $S_{n}$ and $\beta$-decay energies $Q_{\beta}$ of all measured nuclei; these differential quantities are of greater astrophysical relevance than the absolute masses, for both the $r$-process and the inner crust of neutron stars. It will be seen that all models fit the $S_{n}$ better than they fit the absolute masses. However, this is the case for the $Q_{\beta}$ only with model HFB-18. Overall, all four models give mass fits of very similar quality. Likewise, from lines 9 and 10 we see that all four models are essentially equivalent from the standpoint of charge radii (the data are taken from Ref. [25]). The last line of Table III shows the calculated values of the neutron-skin thickness of ${ }^{208} \mathrm{~Pb}$; the experimental values are discussed in Sec IIIB.

Using the forces BSk19, BSk20 and BSk21 we have constructed complete mass tables HFB-19, HFB-20 and HFB-21, respectively, running from one drip line to the other over the range $Z$ and $N \geq 8$ and $Z \leq 110$. We plot the differences between these three mass models in Figure 3, where it will be seen that globally they are very similar, with no striking systematic 


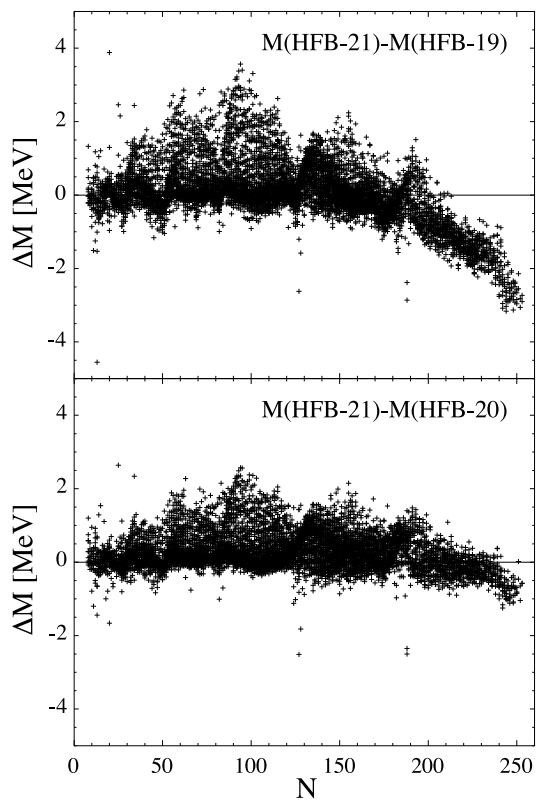

FIG. 3: (Upper panel) Mass differences between HFB-21 and HFB-19 mass models for all 8509 nuclei included in the tables. (Lower panel) Ditto for HFB-21 and HFB-20 models.

differences emerging even for the highest values of $N$, corresponding to the neutron drip line. On the other hand, the upper panel of Figure 4 shows that there are somewhat larger deviations between our models (represented here by the typical HFB-21) and the model D1M [26] based on a Gogny-type force (note the different scale used in Figure 4); moreover, with increasing $N$ there is a systematic tendency for D1M to bind less strongly than the HFB models (the rms deviation of this model for the same data set that we take here is $0.798 \mathrm{MeV}$ ). The lower panel of Figure 4 compares HFB-21 with the FRDM [24], and here we see that at large $N$ the deviations are much bigger than is the case for D1M.

\section{Properties of infinite nuclear matter}

The first seven parameters of Tab. IV are defined by first writing the energy per nucleon of infinite nuclear matter of density $\rho$ and charge asymmetry $\eta=\left(\rho_{n}-\rho_{p}\right) / \rho$ in the form

$$
e(\rho, \eta)=e(\rho, \eta=0)+e_{s y m}^{(1)}(\rho) \eta^{2}+O\left(\eta^{4}\right)
$$

in which the first term on the right-hand side is just the energy per nucleon of SNM; we have neglected charge-symmetry breaking terms, such as those arising from the neutron-proton mass difference (note that in Ref. [4] we wrote $e_{s y m}^{(1)}$ simply as $e_{s y m}$, but here we have to 


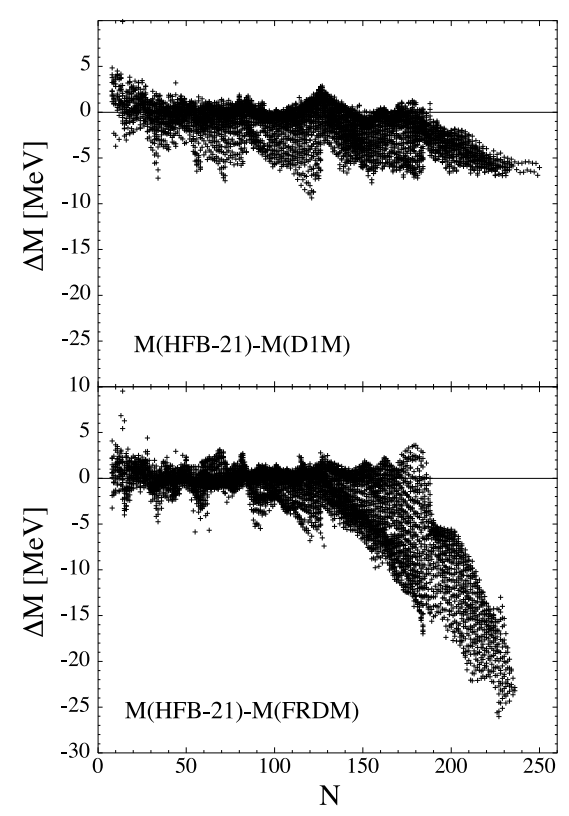

FIG. 4: (Upper panel) Mass differences between HFB-21 and D1M mass models for all 8509 nuclei included in the tables. (Lower panel) Ditto for HFB-21 and FRDM models.

distinguish it from another symmetry energy to be defined in Eq. (11)). We then expand $e(\rho, \eta=0)$ and $e_{s y m}^{(1)}(\rho)$ about the SNM equilibrium density $\rho_{0}$ in powers of $\epsilon=\left(\rho-\rho_{0}\right) / \rho_{0}$, thus

$$
e(\rho, \eta=0)=a_{v}+\frac{1}{18} K_{v} \epsilon^{2}-\frac{1}{162} K^{\prime} \epsilon^{3}+\ldots
$$

and

$$
e_{\text {sym }}^{(1)}(\rho)=J+\frac{1}{3} L \epsilon+\frac{1}{18} K_{\text {sym }} \epsilon^{2}+\ldots \quad .
$$

Since the expressions corresponding to the generalized Skyrme force (2) for the coefficients $K^{\prime}$ and $K_{\text {sym }}$ were not given in the Appendix of Ref. [4] we show them here:

$$
\begin{array}{r}
K^{\prime}=-\frac{12 \hbar^{2}}{5 M} k_{F}^{2}+\frac{3}{8}\left[3 t_{1}+t_{2}\left(5+4 x_{2}\right)\right] \rho k_{F}^{2}-\frac{27}{16}(\alpha+1) \alpha(\alpha-1) t_{3} \rho^{\alpha+1} \\
-\frac{9}{80}(3 \beta+5)(3 \beta+2)(3 \beta-1) t_{4} \rho^{\beta+1} k_{F}^{2} \\
-\frac{3}{80}(3 \gamma+5)(3 \gamma+2)(3 \gamma-1) t_{5}\left(5+4 x_{5}\right) \rho^{\gamma+1} k_{F}^{2}
\end{array}
$$

and

$$
\begin{gathered}
K_{\text {sym }}=-\frac{\hbar^{2}}{3 M} k_{F}^{2}+\frac{5}{12}\left[-3 t_{1} x_{1}+t_{2}\left(4+5 x_{2}\right)\right] \rho k_{F}^{2}-\frac{3}{16} \alpha(\alpha+1) t_{3}\left(1+2 x_{3}\right) \rho^{\alpha+1} \\
-\frac{1}{8}(3 \beta+5)(3 \beta+2) t_{4} x_{4} \rho^{\beta+1} k_{F}^{2}+\frac{1}{24}(3 \gamma+5)(3 \gamma+2) t_{5}\left(4+5 x_{5}\right) \rho^{\gamma+1} k_{F}^{2} .
\end{gathered}
$$


The coefficients appearing in lines 8 and 9 of Table IV are functions of the preceding coefficients, and are defined in Eqs. (14) and (17), respectively. They will be used in Sec III.

Effective masses. The values of the isoscalar and isovector effective masses, $M_{s}^{*}$ and $M_{v}^{*}$, respectively, shown in Table IV] are calculated at the equilibrium density $\rho_{0}$ of SNM. The value $M_{s}^{*}=0.8 M$ for all the models was, as already explained, a constraint imposed on the fit to the data, but the various values of $M_{v}^{*}$ were left free and emerged from the fit. Experimental estimates of this quantity vary widely: Measurements of the isovector giant dipole resonance (IVGDR) in heavy nuclei, as summarized in Figure 47 of Ref. [27], indicate that the value of $M_{v}^{*} / M$ can range from 0.7 to 1 (this figure in effect plots $M / M_{v}^{*}$ ). However, the subsequent discussion in Ref. [27] points out that lower values are possible, which means that none of our models is in clear conflict with experiment. Moreover, all four models have $M_{v}^{*}<M_{s}^{*}$, which implies that the neutron effective mass $M_{n}^{*}$ is larger than the proton effective mass $M_{p}^{*}$ in neutron-rich matter, since the effective mass of a nucleon of charge type $q$ in nuclear matter at density $\rho$ is given by

$$
\frac{M}{M_{q}^{*}}=\frac{2 \rho_{q}}{\rho} \frac{M}{M_{s}^{*}}+\left(1-\frac{2 \rho_{q}}{\rho}\right) \frac{M}{M_{v}^{*}}
$$

This prediction is consistent with measurements of the IVGDR [28], and has been confirmed in many-body calculations with realistic forces [29]. With these latter calculations giving $M_{s}^{*}=0.825 M$ and $M_{v}^{*}=0.727 M$, we see that the magnitude of the splitting given by the new forces, especially BSk21, is much more realistic than that given by BSk18.

Figure 5 shows for the three new models and BSk18 how $M_{s}^{*}$ and $M_{v}^{*}$ vary with density. For Skyrme forces of the conventional form (10) both $1 / M_{s}^{*}$ and $1 / M_{v}^{*}$ depend linearly on the density, as is also the case for BSk18, because of the constraints (3a $)$ - (3c). However, these constraints are not applied to the new forces, BSk19 - BSk21, with the result that $1 / M_{s}^{*}$ and $1 / M_{v}^{*}$ will vary non-linearly with density for these three forces. This accounts for the low-density peak seen in $M_{s}^{*}$ for these forces. There are peaks also in $M_{v}^{*}$ for all three of the new forces, but they occur at such low densities that they are quite invisible in Figure 5 .

Using now Eq. (10) and the density distributions given by the HFB calculations, we calculate the radial variation of $M_{n}^{*}$ and $M_{p}^{*}$ in ${ }^{208} \mathrm{~Pb}$, and show the results for the three new models and BSk18 in Figure 6. We see that the average value of $M_{n}^{*}$ over the nucleus of ${ }^{208} \mathrm{~Pb}$ will be significantly larger for the three new forces than for BSk18, and we would therefore expect the neutron single-particle (s.p.) level density close to the Fermi surface to 


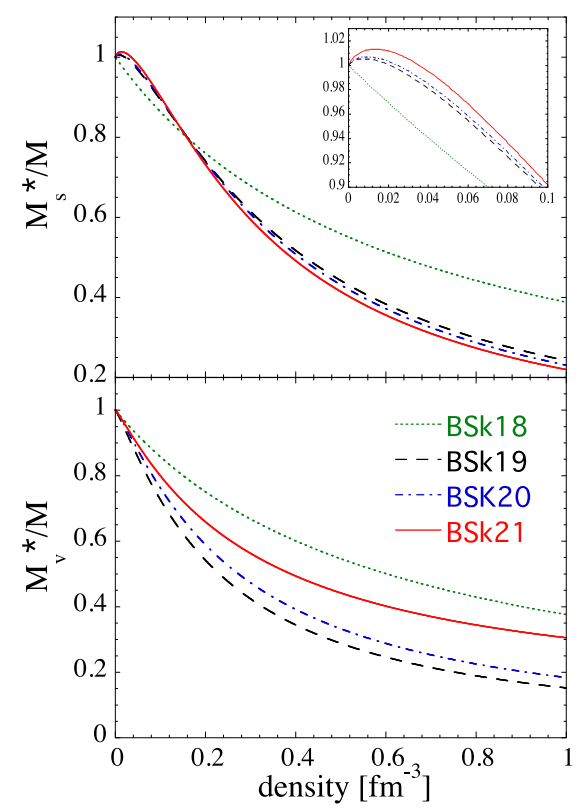

FIG. 5: (Color online) Variation with density of $M_{s}^{*} / M$ (upper panel) and $M_{v}^{*} / M$ (lower panel).

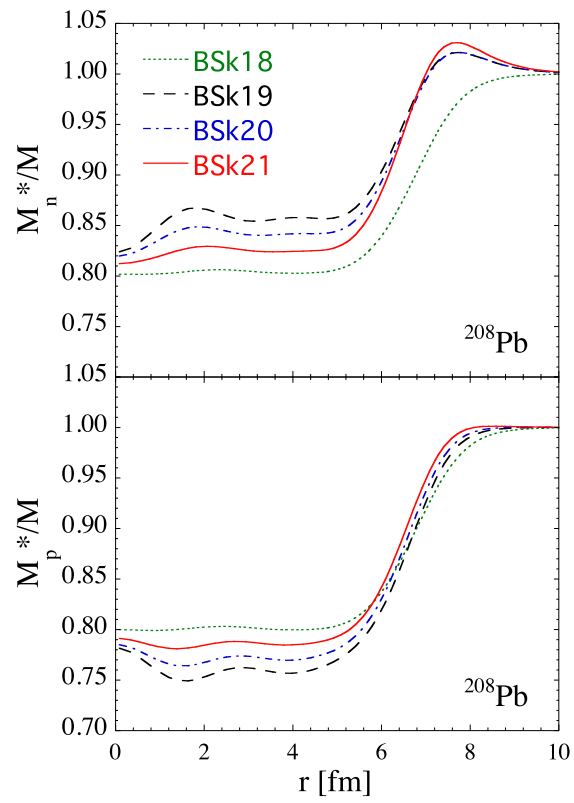

FIG. 6: (Color online) Variation of $M_{n}^{*} / M$ (upper panel) and $M_{p}^{*} / M$ (lower panel) with radial position in ${ }^{208} \mathrm{~Pb}$.

be greater for the new forces than for BSk18. Table $[\mathrm{V}$ shows that this is indeed the case (of the new forces we show only BSk20 in this and Table VI since the other two give almost identical s.p. energies), if we take as a measure of the s.p. level density the separation of certain spin-orbit doublets (we have to adopt this measure because of the distorting effect 
of the spin-orbit splitting). However, for all four forces the neutron s.p. level density is still much lower than the experimental value (our data on s.p. energies are taken from Ref. [30]), essentially because even for the new forces the average value of $M_{n}^{*}$ over the nucleus is not big enough. On the other hand, for the proton s.p. level densities the new forces give just as poor an agreement with experiment as does BSk18 (Table VI), a result that can be understood from the lower panel of Figure 6, where it will be seen that $M_{p}^{*}$ for the new forces lies sometimes above and sometimes below the BSk18 value.

Nevertheless, we see that with a more thorough exploitation of the $t_{4}$ and $t_{5}$ terms it should be possible in principle for the s.p. level densities to be well reproduced, at least in heavy nuclei, while maintaining realistic values of $M_{s}^{*}$ and $M_{v}^{*}$ in SNM at density $\rho_{0}$. Some partial success in this direction has already been achieved in Ref. [31], where the s.p. proton level density in the vicinity of the Fermi surface of ${ }^{208} \mathrm{~Pb}$ was well reproduced, although the s.p. neutron level density was far too low, and no attempt was made to impose a global mass fit. The peaks in that work were much stronger than here, $M_{s}^{*}$ having a maximum value of $1.13 M$, while $M_{v}^{*}$ had a maximum value of $1.33 M$, suggesting that forces BSk19BSk21 do not have nearly enough non-linearity in $1 / M_{s}^{*}$ and $1 / M_{v}^{*}$. However, sufficiently strengthening the peaks in $M_{s}^{*}$ and $M_{v}^{*}$ while maintaining the present quality of the mass fits and respecting all the other constraints might be impossible with the present form (2) of Skyrme force: despite its great generality it might have to be generalized still further by adding extra $t_{3}, t_{4}$ and $t_{5}$ terms, each characterized by different density exponents. Fitting such a force would be highly labor intensive.

Distribution of potential energy among the $(S, T)$ channels. Fitting our forces to the mass data and the EOS of NeuM is not a sufficient condition for ensuring a realistic distribution of the potential energy per nucleon among the four two-body spin-isospin $(S, T)$ channels in SNM. This is made clear in Figure [7, which shows this distribution in SNM for each of our three new forces, calculated using Eq. (B6) of Ref. [28], as a function of density, and compares with two different BHF calculations: "Catania 1", based on Ref. [8] and "Catania 2", based on Ref. [32]. The data of the former were kindly supplied by U. Lombardo [33] and of the latter by M. Baldo [34]. Given the evident uncertainty in what the real distribution actually is, the level of agreement we have found with our new forces can be regarded as satisfactory. This is the first time that we have been able to achieve such a level of conformity to reality with any of our forces; the improvement in the $(1,1)$ channel is particularly striking, as 


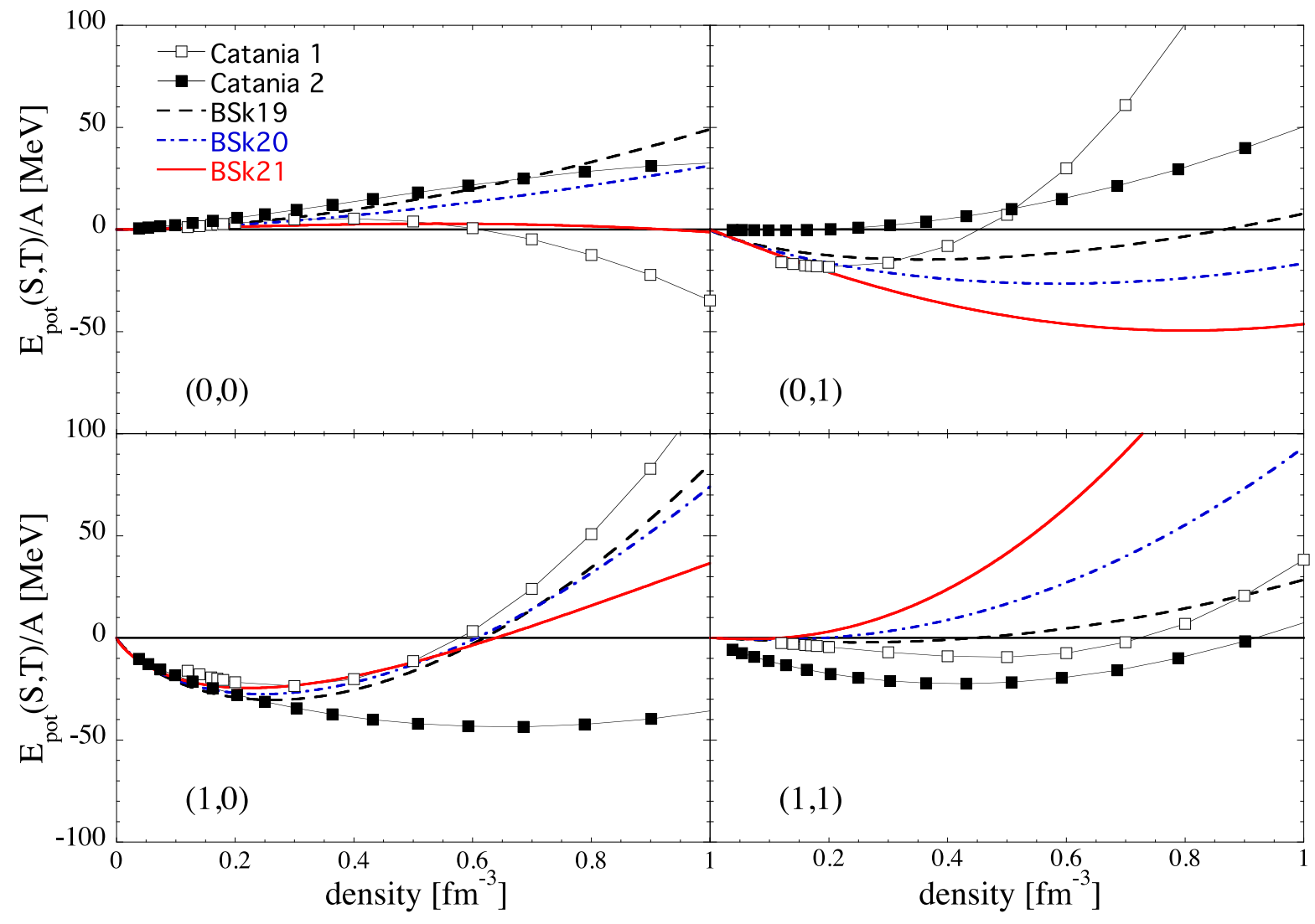

FIG. 7: (Color online) Potential energy per particle $E_{\text {pot }} / A$ in each $(S, T)$ channel as a function of density for symmetric infinite nuclear matter. The open symbols correspond to the "Catania 1" BHF calculations [8], and the solid symbols to the "Catania 2" BHF calculations [32].

can be seen by comparing Figure 7 with Figure 4 of Ref. [1] and Figure 9 of Ref. [11]. As discussed in Refs. [11] and [28], this improvement would have been very difficult within the framework of conventional Skyrme forces, and the terms in $t_{4}$ and $t_{5}$ have been indispensable in this respect.

\section{SYMMETRY ENERGY}

\section{A. Generalities}

The properties of neutron-star matter $\left(\mathrm{N}^{*} \mathrm{M}\right)$ depend crucially on the difference between the energy per nucleon in NeuM and the energy per nucleon in SNM, i.e., on the symmetry 


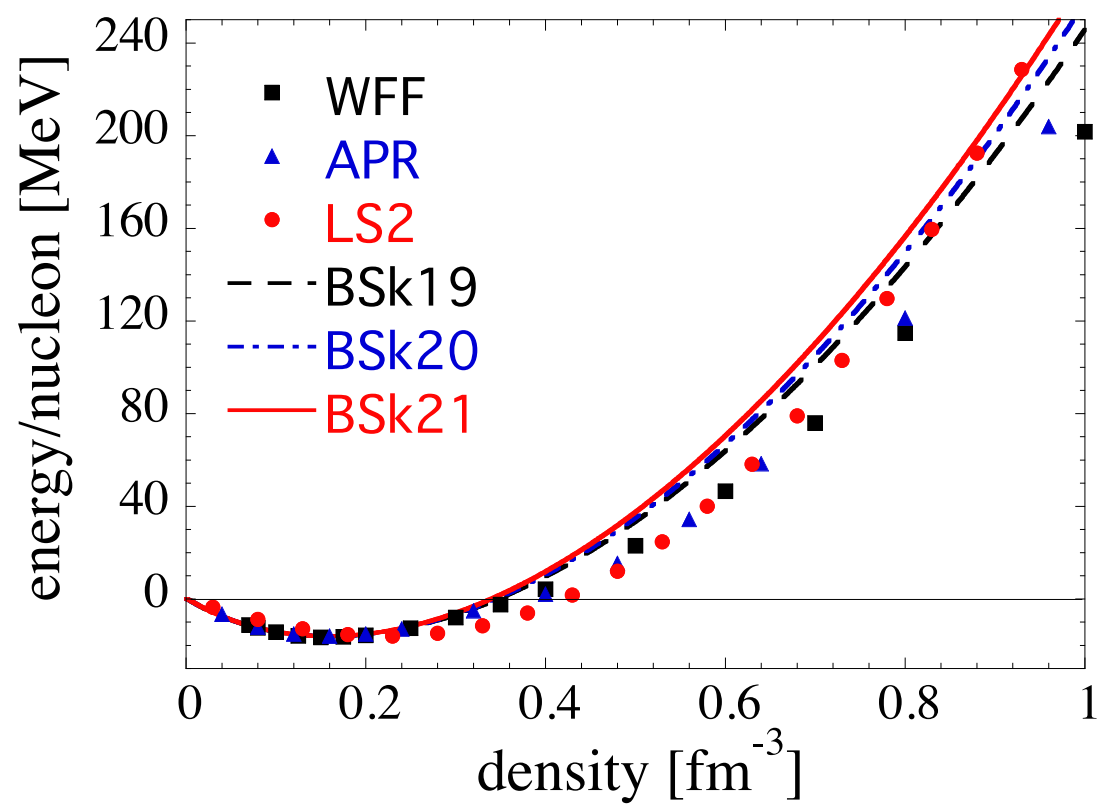

FIG. 8: (Color online) Zero-temperature EOSs for symmetric nuclear matter (SNM) with forces BSk19 - BSk21. Also shown are the realistic EOSs WFF [22], APR [6] and LS2 [8].

energy

$$
e_{\text {sym }}^{(2)}(\rho)=e(\rho, \eta=1)-e(\rho, \eta=0)
$$

(see Sec V). Because of quartic and higher-order terms in $\eta$ this symmetry energy is not identical to the symmetry energy $e_{\text {sym }}^{(1)}(\rho)$ defined in Eq. (77) [35]; we return to this point later in this section. Figure 8 shows that the EOSs in SNM for our three new forces, BSk19 - BSk21, are remarkably similar, despite having quite different properties in NeuM (see also Figure 2). It seems that the fit to the data, none of which relates to densities much greater than $\rho_{0}$, determines almost completely the properties of SNM up to more than $4 \rho_{0}$, at least under the constraint of given values of $J$ and $K_{v}$. Moreover, we have calculated the pressure in SNM as a function of density for our forces and find that it is consistent with measurements of nuclear-matter flow in heavy-ion collisions, although close to the upper limit: see Figure 3 of Ref. [36].

In Figure 8 we show also the realistic EOSs for SNM given by the WFF [22], APR [6] and LS2 [8] calculations. It will be seen that while BSk19 agrees very closely (by construction) with WFF in NeuM (and thus with FP [5]), it is significantly stiffer in SNM; a similar remark applies to BSk20 and its APR counterpart, and likewise to BSk21 and its LS2 counterpart. In this connection it should be realized that the uncertainties in many-body theory are even 


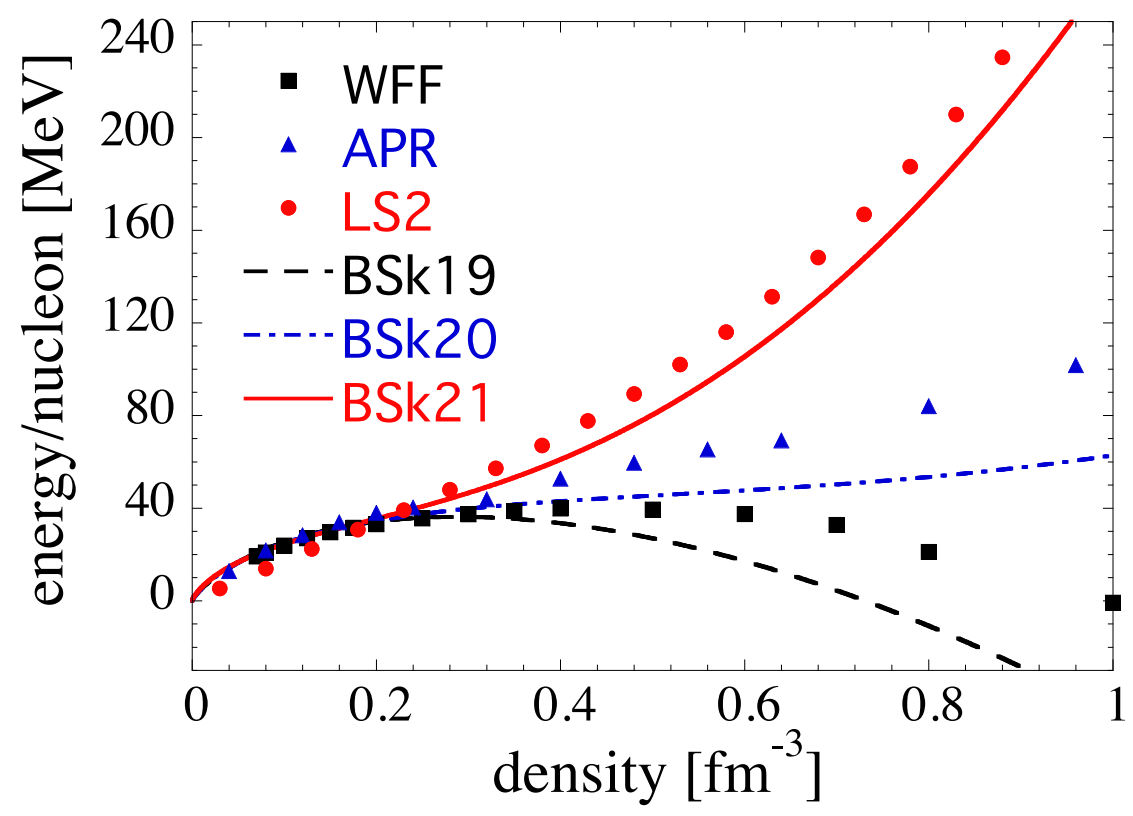

FIG. 9: (Color online) Symmetry energy $e_{s y m}^{(2)}$ for forces BSk19, BSk20 and BSk21. Also shown are the realistic EOSs WFF [22], APR [6] and LS2 [8].

greater for SNM than for NeuM, essentially because of the ${ }^{3} S_{1}-{ }^{3} D_{1}$ tensor coupling, which acts in the former but not the latter. Thus in all such calculations performed so far it has been necessary to make a phenomenological adjustment of the three-body force in order to get an acceptable equilibrium point of SNM. The way in which this adjustment is made is far from unique, both with regard to the parametrization of the three-body force and the actual values of the parameters $a_{v}$ and $\rho_{0}$ characterizing the equilibrium point of SNM (we stress that in the case of our forces the values of $a_{v}$ and $\rho_{0}$ given in Table IV were not fixed a priori but emerged from the mass fit).

Using Figs. 1 and 8, we now plot in Figure 9 the symmetry energy $e_{s y m}^{(2)}$ for all the forces of Figure 8. We see that our three forces agree very closely in their predictions for $e_{s y m}^{(2)}$ up to and slightly beyond $\rho_{0}$. However, as the density increases the curves for our forces diverge strongly from each other. In fact, for BSk19 $e_{\text {sym }}^{(2)}$ becomes negative at high densities, NeuM having lower energy per nucleon than SNM. Figure 9 also shows that WFF (and thus FP) has higher symmetry energy than its BSk19 counterpart, and likewise for APR as compared to BSk20, and LS2 as compared to BSk21. These differences can be traced almost entirely to the differences in SNM that we have remarked above (note that stiffer SNM implies softer symmetry energy, for a given EOS of NeuM). 


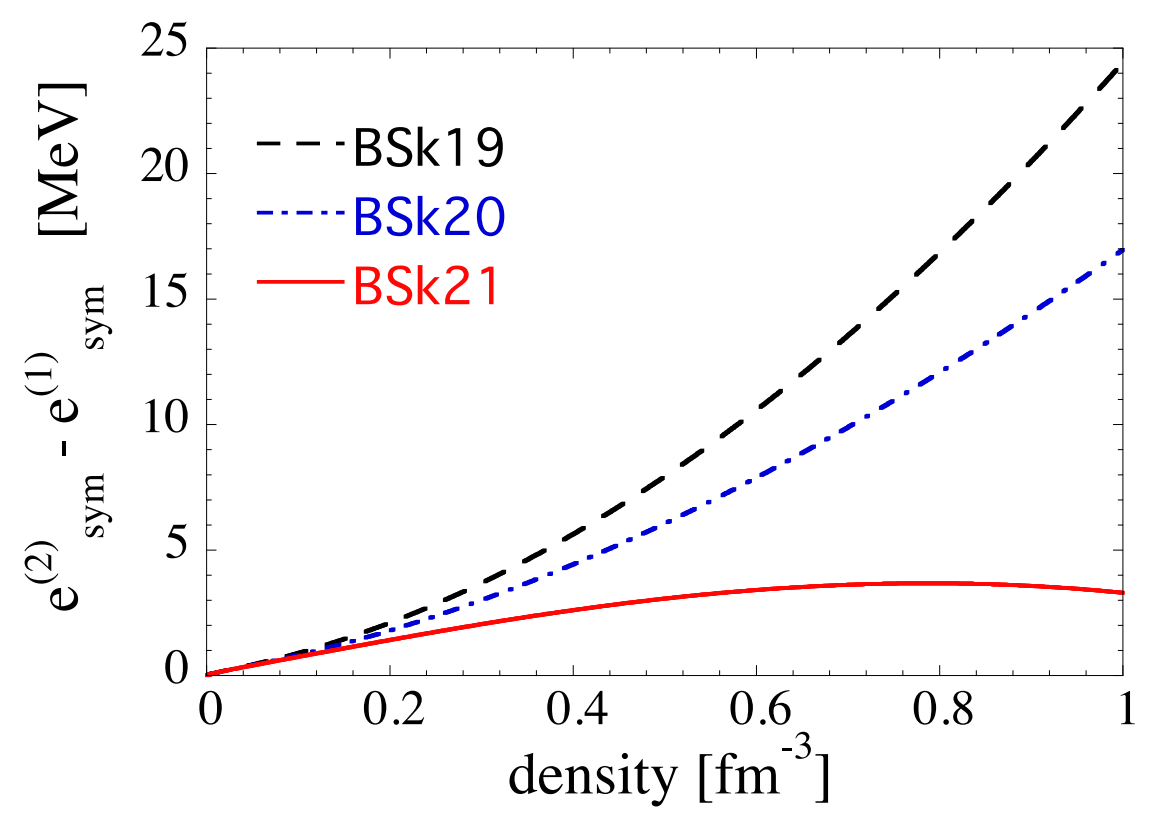

FIG. 10: (Color online) Difference $e_{s y m}^{(2)}-e_{s y m}^{(1)}$ for forces BSk19 - BSk21.

To examine the relationship between the symmetry energy $e_{s y m}^{(2)}$ discussed in this section and the symmetry energy $e_{s y m}^{(1)}$ defined in Eq. (7) we plot in Figure 10 the difference $e_{s y m}^{(2)}-$ $e_{s y m}^{(1)}$ for our three new forces. Contrary to the usual assumption, this difference is seen to be far from negligible, amounting to around $1 \mathrm{MeV}$ in the vicinity of $\rho=\rho_{0}$, and possibly becoming much larger at higher densities, depending on the force in question.

High-density behavior. Since the EOSs of NeuM to which we have fitted our three new forces differ so strongly at high densities, it would be highly desirable to know how the symmetry energy actually does vary with density at high density. Unfortunately, as we now see, our knowledge in this respect is extremely limited. Turning first to many-body theory with realistic forces, we note that all three of our new forces are supported by their realistic counterparts. In particular, the plausibility of the negative symmetry energy of BSk19 is supported by the realistic EOS of WFF. The reason why the realistic calculations of the symmetry energy show almost as large a high-density divergence as our phenomenological forces lies at least partially with the choice of three-nucleon force and its behavior at high densities. Dirac-Brueckner-Hartree-Fock calculations, in which there is no three-nucleon force, also show a high-density softening of the EOS [37].

In the present state of the theory only experiment can decide the issue, but so far there has been only one measurement of symmetry energy at the high densities found in the core 
of neutron stars. This involves pion production in heavy-ion collisions at very high energies, measuring the $\pi^{-} / \pi^{+}$ratio [7]. It was found that the data were consistent with models predicting negative symmetry energy at densities above $3 \rho_{0}$.

Low-density behavior. Since $e_{\text {sym }}^{(2)}$ is roughly equal to $e_{\text {sym }}^{(1)}$ for our forces at nuclear and lower densities it can be represented by the expansion (8b) in this density region, at least qualitatively. The close agreement between our forces seen in Figure 9 for these densities can now be related firstly to the fact that $J$ is the same for all our forces (by choice), and also to the fact that the $L$ coefficient, which measures the slopes of the curves at $\rho=\rho_{0}$, has similar values, as shown in Table IV. Moreover, the origin of the high-density divergence between our EOSs can be found in Table IV, where we see that the second derivative of these curves, measured by $K_{\text {sym }}$, differs considerably from one force to another.

We devote the rest of this section to our knowledge, both experimental and theoretical, of symmetry energy in the region of nuclear densities, since much more information is available here than in the high-density region.

\section{B. The $J$ and $L$ coefficients}

The determination of the $J$ and $L$ coefficients from measurements on finite nuclei requires the use of some model to describe the nuclei in question. One obvious approach is via mass measurements, but fits of mass models to the data yield a wide range of values of $J$ and $L$. For example, the finite-range droplet model [24] yields $J=32.73 \mathrm{MeV}$ (actually, this is just for the macroscopic part; in the microscopic part $J$ is given as $35 \mathrm{MeV}$ ). On the other hand, mass models based on Skyrme forces of the conventional form (11) give an optimal mass fit with $J \approx 27.5 \mathrm{MeV}$ [13] (we adopt higher values in all our HFB models in order to avoid a collapse of NeuM, which would otherwise occur even when assuming a non-polarized ground state). However, despite this ambiguity in the value of $J$ given by mass fits there is a fairly tight correlation with the value of $L$. This correlation was explored more than 30 years ago [38], and then in Ref. [39] (see especially Table 2 of that article); it has subsequently been revisited several times, e.g., Refs. [40 42]. As for our own HFB mass models, the $L-J$ correlation is clearly seen in Table 3 of Ref. [13], and is manifested in the present article by the relative closeness of the $L$ values for our forces.

The fact that fitting nuclear masses correlates $J$ with $L$ can be easily understood as 
follows. Increasing $J$ will increase the symmetry energy in the center of the nucleus, an increase that will have to be compensated by a decrease in the symmetry energy in the surface. Such a decrease can be achieved by a reduction in $L$, since $\rho<\rho_{0}$ in that region.

A similar but somewhat weaker $L-J$ correlation is also found in measurements of isospin diffusion in heavy-ion collisions: see Figure 3 of Tsang et al. [43]. The data leave a large range in the possible values of $L$ for a given value of $J$, but for our chosen value of $J=30$ $\mathrm{MeV}$ the lower limit on $L$ is about $45 \mathrm{MeV}$, which is consistent with force BSk21, but not the other forces.

We now summarize two types of measurement that lead in principle to unique values of either $J$ or $L$.

Heavy-ion collisions. Chen et al. [44] have analyzed isospin-diffusion data and find $L=$ $88 \pm 25 \mathrm{MeV}$, which is much higher than is given by any of our forces. On the other hand, the analysis of Famiano et al. [45] finds that the symmetry energy varies roughly as $\rho^{1 / 2}$, which yields $L \approx 3 J / 2$, i.e., $47 \pm 2 \mathrm{MeV}$, which agrees best with BSk21. The interpretation of these experiments is clearly very model dependent.

Neutron-skin thickness. For a given nucleus this is defined by

$$
\theta \equiv R_{n}^{r m s}-R_{p}^{r m s}
$$

where the rms radii refer to point nucleons. By considering the results of non-relativistic Hartree-Fock and relativistic mean-field calculations with many different forces it was noted by Brown [46] and by Typel and Brown [47] that a strong correlation exists between the neutron-skin thickness and the value of the $L$ coefficient. Thus the strong correlation already noted between $L$ and $J$ implies that the value of $\theta$ is likewise correlated with $J$ : see, for example, Table 2 of Ref. [39]. This correlation can easily be understood [13] in terms of the droplet-model expression (2.21) of Ref. [48] for the neutron-skin thickness of a nucleus of atomic number $Z$ and mass number $A$,

$$
\theta=\frac{3}{2} r_{0} \frac{J}{Q} I
$$

where $r_{0}=\left(3 / 4 \pi \rho_{0}\right)^{1 / 3}, I=(N-Z) / A$ and $Q$ is the surface-stiffness coefficient, which is anticorrelated with $J$ if masses are fitted [49, 50]; see also Ref. [41] for a recent extensive discussion. (The correlation between $\theta$ and $J$ might be expected to be more robust than that between $\theta$ and $L$, since the latter can hold only to the extent that a local-density approximation is valid in the surface, $L$ relating to homogeneous nuclear matter.) 
Measurement of the neutron-skin thickness is difficult, but one particular method involves the use of antiprotons as a probe [51]. Analyzing the results on 26 nuclei, Ref. [41] found $L=55 \pm 25 \mathrm{MeV}$, which is compatible with all three forces of this article.

A nucleus of particular interest is ${ }^{208} \mathrm{~Pb}$, since several measurements of its neutron-skin thickness have been made, and a very precise one is being planned. It is for this reason that we show in the last line of Table III our calculated values of the neutron-skin thickness for this nucleus. One of the lowest published values, obtained from high-energy proton scattering, is $0.14 \pm 0.04 \mathrm{fm}$ [52], which certainly agrees with all our forces. A similarly low value, $0.15 \pm 0.02 \mathrm{fm}$, was originally extracted from the antiprotonic measurements [51], but a new analysis of this data [53] gives a result that is barely consistent with any of our forces: $0.20 \pm 0.04 \pm 0.05 \mathrm{fm}$., in which the first error bar represents the experimental uncertainty and the second the theoretical uncertainty associated with the strong-interaction model.

This latter source of uncertainty besets, in one form or another, all the different determinations of neutron-skin thickness published so far, but will be avoided in the proposed PREX experiment [54], which is to measure parity violation in the elastic scattering of electrons on ${ }^{208} \mathrm{~Pb}$. However, the expected error bars, $\pm 0.05 \mathrm{fm}$., will still be much too large to distinguish between our different forces. On the other hand, it is conceivable that the PREX experiment will give a result that is quite incompatible with any of our predictions. In that case it would be necessary to make a new mass fit with $J$ constrained to a value appropriately higher or lower than the value of $30 \mathrm{MeV}$ taken here.

Many-body theory with realistic forces. Several such calculations of $J$ and $L$ have been published, but usually these consider only SNM and NeuM, and then assume that $e_{\text {sym }}^{(1)}=$ $e_{\text {sym }}^{(2)}$, an approximation that we estimate, on the basis of the forces BSk18-21, to lead to $J$ being overestimated by around $1 \mathrm{MeV}$. Further errors in the values of $J$ and $L$ calculated in this way arise from the uncertainties that are specific to the many-body calculation of SNM (see above). Thus it is not surprising that the values of $J$ and $L$ predicted by different calculations should disagree widely, as we now see.

For example, while the three BHF calculations of Ref. [55] have $J$ lying in the range 34.7 $\pm 1.1 \mathrm{MeV}$ and $L$ in the range $65.0 \pm 1.9 \mathrm{MeV}$, the BHF calculations underlying the LS2 EOS (to which BSk21 was constrained) yield the quite different values of $J=30.6 \mathrm{MeV}$ and $L=101.4 \mathrm{MeV}$ [8]. Our final example is the BHF calculation [8] using the Bonn B [56] two-nucleon interaction and a three-nucleon interaction of the same realistic form as adopted 
for LS2: this calculation gives $J=29.4 \mathrm{MeV}$ and $L=74.4 \mathrm{MeV}$.

All in all, it is clear that in the search for the correct values of $J$ and $L$ many-body theory does not help us to narrow down the wide range of possibilities left open by experiment.

\section{The $K_{\text {sym }}$ coefficient}

The coefficient $K_{\text {sym }}$ appearing in the expansion (8b) can only be measured in the combination

$$
K_{\tau}=K_{\text {sym }}+L\left(\frac{K^{\prime}}{K_{v}}-6\right),
$$

because for non-zero $L$-values the equilibrium density of asymmetric nuclear matter is displaced away from $\rho_{0}$.

Many-body theory with realistic forces. The only such calculations of $K_{\tau}$ with which we are familiar are the three BHF calculations of Ref. [55], which yield values lying in the range -344 to -335 MeV, discriminating thereby against BSk21. Of course, the doubts we expressed above concerning the reliability of calculations of $J$ and $L$ with realistic forces apply equally well to these calculations of $K_{\tau}$.

Isospin diffusion in heavy-ion collisions. These measurements yield a value for $K_{\tau}$ of $-370 \pm 120 \mathrm{MeV}$ [57], which is consistent with all our forces (see Table IV), and with the

ab initio calculations of Ref. [55]. Clearly, improved accuracy will be necessary before it is possible to discriminate between our different forces on this basis.

Isotopic variation of "breathing-mode" energies. The coefficient $K_{\tau}$ can in principle be determined by measuring the finite-nucleus incompressibility $K(Z, A)$ of a string of isotopes of the same element. Such a determination was made recently in Ref. [58] by measurements of the energies of the giant isoscalar monopole resonance $E_{G M R}$ in the seven even-even $\mathrm{Sn}$ isotopes between ${ }^{112} \mathrm{Sn}$ and ${ }^{124} \mathrm{Sn}$. Using the relation

$$
K(Z, A)=\frac{M}{\hbar^{2}} R^{2} E_{G M R}^{2},
$$

where $\mathrm{R}$ is the rms matter radius, they fitted their results to the simple expression [59]

$$
K(Z, A)=K_{v}+K_{s f} A^{-1 / 3}+K_{\tau} I^{2}+K_{\text {coul }} \frac{Z^{2}}{A^{4 / 3}},
$$

in which

$$
K_{\text {coul }}=\frac{3 e^{2}}{5 r_{0}}\left(\frac{K^{\prime}}{K_{v}}-8\right)
$$


The parametrization (16), which assumes a scaling model for the resonance, is based on the leptodermous approximation [48] and makes use of the expansions (8a) and (8b) for infinite nuclear matter, only the lowest-order terms beyond $K_{v}$ being retained. Likewise, only the lowest order associated with semi-infinite nuclear matter, $K_{s f} A^{-1 / 3}$, is retained.

The value of $K_{\tau}$ that Ref. [58] extracts from the measurements is $-550 \pm 100 \mathrm{MeV}$, in clearcut disagreement with all our forces (line 8 of Table IV), and with the ab initio calculations of Ref. [55]. It also barely overlaps with the measurement of Ref. [57]. Moreover, it has been argued [60, 61] that a value $K_{\tau} \approx-550 \mathrm{MeV}$ is too strongly negative to be compatible with the behavior of low-density neutron matter, which is determined unambiguously by low-energy neutron-neutron scattering.

However, as we have recently pointed out [62], the analysis that Ref. [58] made of their data is invalid, essentially because as soon as higher-order terms, notably the surfacesymmetry term $K_{s s} I^{2} A^{-1 / 3}$, are admitted into the leptodermous expansion of $K(A, Z)$ then $K_{\tau}$ becomes indeterminate, and it is impossible to say that values in the vicinity of -350 $\mathrm{MeV}$ are not consistent with the data (see also Colò [63]). Actually, the situation becomes even worse when one realizes that it is not possible to identify the $K_{v}$ of Eq. (16) with the incompressibility of infinite nuclear matter $K_{\infty}$ if the structure of the breathing mode deviates from a strict scaling model [64, 65]; similar considerations must also apply to $K_{\tau}$ itself, since it refers, like $K_{v}$, to a volume term. The only reliable way to see whether or not our forces are consistent with the breathing-mode data would be to use them in self-consistent QRPA (or constrained HFB) calculations of the breathing-mode energies [65], which is beyond the scope of the present article.

This approach has, in fact, been followed by Piekarewicz [61, 66], whose RPA calculations are based on the relativistic mean-field method, using the FSU Gold parameters [67]. This interaction reproduces well a certain number of key data points, and in particular the measured breathing-mode energies of ${ }^{208} \mathrm{~Pb},{ }^{144} \mathrm{Sm}$, and ${ }^{90} \mathrm{Zr}$. On the other hand, his calculated breathing-mode energies for the Sn isotopes lie significantly higher than the measured values of Ref. [58]. The value of $K_{\tau}$ for FSU Gold is $-276.8 \mathrm{MeV}$, very close to our value for interaction BSk20, and differing insignificantly from the values for the two other forces of this article.

The two attempts that have been made to account for the anomalously soft properties of the Sn isotopes are summarized in Ref. [61], and found to be incomplete: we are left with an 
open problem in nuclear structure. As far as the present article is concerned the situation is rather troubling, since as long as one does not understand the compressional properties of the $\mathrm{Sn}$ isotopes one cannot claim to fully understand those of the other nuclei from which values of $K_{v}$ are extracted. The most that we can conclude is that at the present time the breathing-mode measurements offer no basis for eliminating any of the forces discussed here. On the other hand, if it turns out that the solution to the tin problem lies in taking a value for $K_{v}$ that differs radically from what we have assumed here the present forces would all have to be abandoned and new fits made.

\section{LANDAU PARAMETERS AND STABILITY CONDITIONS}

The values of the dimensionless Landau parameters in SNM at the equilibrium density $\rho_{0}$ are given in lines $12-19$ of Table IV. All four forces predict almost identical values of the parameters $F_{0}, F_{0}^{\prime}$ and $F_{1}$, even though these parameters can in principle be modified by the new $t_{4}$ and $t_{5}$ terms (except in the case of force BSk18, where the conditions (3a) - (3c) are imposed). The close agreement between the different forces can be traced back to the constraints imposed during the mass fit on the incompressibility $K_{v}$, the isoscalar

effective mass $M_{s}^{*}$ and the symmetry energy $J$, since these quantities are related uniquely to the Landau parameters through

$$
\begin{gathered}
K_{v}=\frac{3 \hbar^{2} k_{F}^{2}}{M_{s}^{*}}\left(1+F_{0}\right), \\
\frac{M_{s}^{*}}{M}=1+\frac{F_{1}}{3}
\end{gathered}
$$

and

$$
J=\frac{\hbar^{2} k_{F}^{2}}{6 M_{s}^{*}}\left(1+F_{0}^{\prime}\right)
$$

However the four forces yield quite different predictions for the remaining Landau parameters. The differences in the values of $F_{1}^{\prime}$ can easily be understood in terms of the different values of the isovector effective mass, since the two quantities are related by

$$
\frac{M_{s}^{*}}{M_{v}^{*}}=1+\frac{F_{1}^{\prime}}{3} .
$$


As for the parameters $G_{0}, G_{0}^{\prime}, G_{1}$, and $G_{1}^{\prime}$, it will be seen that the three new forces give values that differ substancially from those of the older BSk18 force. In particular, $G_{1}$ and $G_{1}^{\prime}$ are now identically zero, while $G_{0}$ and $G_{0}^{\prime}$ are significantly higher. This is a result of dropping the time-odd terms associated with the neglect of the $J^{2}$ and $J_{q}^{2}$ terms (see Sec 【A). Our value of $G_{0}^{\prime}, 0.95$ for all three new forces, is in good agreement with the value of around 1.2 found in BHF calculations using the Argonne A18 potential with a three-body force [68]. (On the other hand, all our values of $G_{0}$ are significantly lower than the value of around 0.8 found in these same realistic calculations, although some improvement over BSk18 can be seen in the three new models.) Our value of $G_{0}^{\prime}$ also falls within the empirical range of $1.0 \pm 0.1$ deduced in Ref. [69] from the analysis of Gamow-Teller (GT) resonances and magnetic-dipole modes in finite nuclei.

It is well-known that for the Fermi liquid theory to be internally consistent, the Landau parameters have to satisfy the two basic sum rules which follow from the Pauli exclusion principle [70]

$$
\begin{aligned}
& S_{1}=\sum_{\ell} \frac{F_{\ell}}{1+F_{\ell} /(2 \ell+1)}+\frac{F_{\ell}^{\prime}}{1+F_{\ell}^{\prime} /(2 \ell+1)} \\
& +\frac{G_{\ell}}{1+G_{\ell} /(2 \ell+1)}+\frac{G_{\ell}^{\prime}}{1+G_{\ell}^{\prime} /(2 \ell+1)}=0
\end{aligned}
$$

and

$$
\begin{gathered}
S_{2}=\sum_{\ell} \frac{F_{\ell}}{1+F_{\ell} /(2 \ell+1)}-3 \frac{F_{\ell}^{\prime}}{1+F_{\ell}^{\prime} /(2 \ell+1)} \\
-3 \frac{G_{\ell}}{1+G_{\ell} /(2 \ell+1)}+9 \frac{G_{\ell}^{\prime}}{1+G_{\ell}^{\prime} /(2 \ell+1)}=0 .
\end{gathered}
$$

Skyrme forces generally violate these sum rules. However, we have found that our new forces significantly improve the second sum rule compared to our previous forces. In particular, while for BSk18 we have $S_{2}=3.05$, our new forces BSk19, BSk20 and BSk21 yield -0.13, -0.26 and -0.03 , respectively. In the latter case, the discrepancy between the calculated value of $S_{2}$ and the exact result is reduced by two orders of magnitude. On the other hand, the values of the first sum $S_{1}$ for the different forces remain very close, lying between 0.7 and 0.9. All the forces quoted above include $t_{4}$ and $t_{5}$ terms. For comparison, our parametrization BSk17 based on a conventional Skyrme force yields $S_{1}=-0.76$ and $S_{2}=7.42$.

Extending the calculation of the Landau parameters to higher densities we find, with one exception, that for all densities $\rho>\rho_{0}$ encountered in the interior of supernova cores and 
neutron stars, the new forces satistify the following stability conditions for $\ell=0$ and 1 , the only values applicable in the case of Skyrme forces:

$$
F_{\ell}>-(2 \ell+1) \quad, \quad F_{\ell}^{\prime}>-(2 \ell+1)
$$

and

$$
G_{\ell}>-(2 \ell+1) \quad, \quad G_{\ell}^{\prime}>-(2 \ell+1)
$$

as shown in Figures 11)(these conditions are still fulfilled when the time-odd terms associated with $J^{2}$ and $J_{q}^{2}$ terms are taken into account). The $\ell=0$ cases are of particular interest, as we now discuss.

(i) The condition on $G_{0}$ ensures that the spin asymmetry coefficient, defined by

$$
a_{\sigma} \equiv\left(\frac{1}{2} \frac{\partial^{2} e}{\partial I_{\sigma}^{2}}\right)_{I_{\sigma}=0}=\frac{\hbar^{2} k_{F}^{2}}{6 M_{s}^{*}}\left(1+G_{0}\right)
$$

where $I_{\sigma}=\left(\rho_{\uparrow}-\rho_{\downarrow}\right) / \rho$, always remains positive, i.e., SNM is stable with respect to a ferromagnetic transition.

(ii) The condition on $G_{0}^{\prime}$ ensures that the spin-isospin asymmetry coefficient, defined by

$$
a_{\sigma \tau} \equiv \frac{1}{2}\left(\frac{\partial^{2} e}{\partial I_{\sigma \tau}^{2}}\right)_{I_{\sigma \tau}=0}=\frac{\hbar^{2} k_{F}^{2}}{6 M_{s}^{*}}\left(1+G_{0}^{\prime}\right),
$$

where $I_{\sigma \tau}=\left(\rho_{n \uparrow}-\rho_{n \downarrow}-\rho_{p \uparrow}+\rho_{p \downarrow}\right) / \rho$, always remains positive, i.e., there is no instability with respect to spin-isospin transitions. Unlike our three new forces, BSk19, BSk20 and BSk21, our earlier model force BSk18 predicts that such an instability occurs at density of $\rho=0.62 \mathrm{fm}^{-3}$.

(iii) The condition on $F_{0}$ ensures that the incompressibility coefficient, defined by

$$
a_{\rho} \equiv \frac{1}{2} \frac{\partial^{2} e}{\partial\left(\rho / \rho_{0}\right)^{2}}=\frac{\hbar^{2} k_{F}^{2}}{6 M_{s}^{*}}\left(1+F_{0}\right),
$$

is always positive, at least for $\rho>\rho_{0}$, i.e., SNM is stable against mechanical breakup, of the sort that occurs for $\rho<\rho_{0}$. [Note that $a_{\rho}\left(\rho=\rho_{0}\right)=K_{v} / 18 \rho_{0}^{2}$, whence Eq. (18a) follows.]

(iv) The condition on $F_{0}^{\prime}$, where it holds, ensures that the isospin asymmetry coefficient, defined by

$$
a_{\tau} \equiv \frac{1}{2}\left(\frac{\partial^{2} e}{\partial I_{\tau}^{2}}\right)_{I_{\tau}=0}=\frac{\hbar^{2} k_{F}^{2}}{6 M_{s}^{*}}\left(1+F_{0}^{\prime}\right)
$$



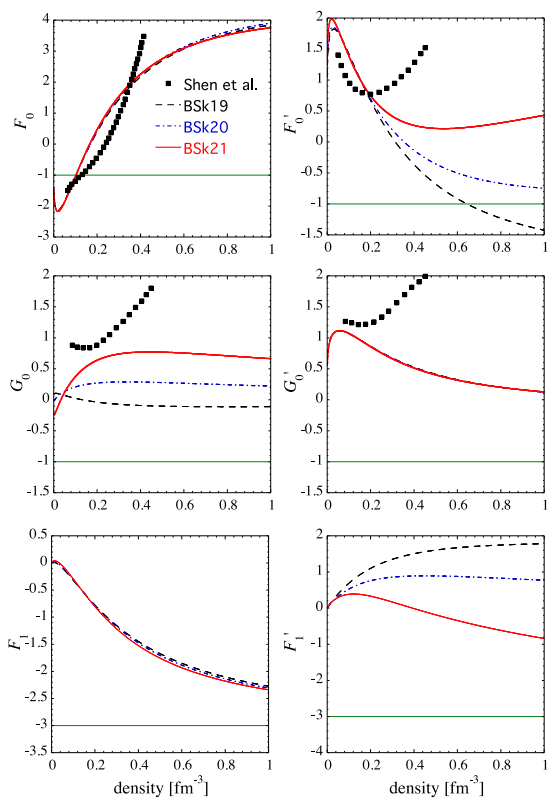

FIG. 11: (Color online) Landau parameters in symmetric nuclear matter for forces BSk19 - BSk21 (note that the curves for the three forces are indistinguishable from each other in the case of $F_{0}, F_{1}$ and $G_{0}^{\prime}$ ). For comparison we show also the results of BHF calculations from Ref. [1]]. We also indicate the stability limit $-(2 \ell+1)$.

where $I_{\tau}=\left(\rho_{n}-\rho_{p}\right) / \rho$, always remains positive, i.e., there is no isospin instability, in which the neutrons of SNM tend to become protons, and vice versa. This holds at all densities $\rho>\rho_{0}$ for forces BSk20 and BSk21, but breaks down for BSk19 above $\rho=0.64 \mathrm{fm}^{-3}$, which is precisely the density at which $e_{s y m}^{(1)}$ goes negative for this force. (This is close to, but not coincident with, the density at which $e_{s y m}^{(2)}$ goes negative for BSk19, $0.73 \mathrm{fm}^{-3}$, as can be read off from Figure 9.) There is a similar instability for BSk18.

In addition to instabilities in $F_{0}^{\prime}$ and $G_{0}^{\prime}$, BSk18 predicts instability with respect to fluctuations in the spin-orbit current $\boldsymbol{J}$, since $G_{1}$ falls below -3 at density $\rho=0.79 \mathrm{fm}^{-3}$.

We have also evaluated the Landau parameters in NeuM. The appropriate expressions for the generalized Skyrme force (2) can be found in Appendix B, while the numerical values are shown in Figures 12. As in the case of SNM, all the new forces BSk19 - BSk21 are stable with respect to all the Landau parameters in NeuM. On the other hand, our earlier force BSk18 predicted an instability in $G_{1}^{\text {NeuM }}$ at density $\rho=0.90 \mathrm{fm}^{-3}$. 

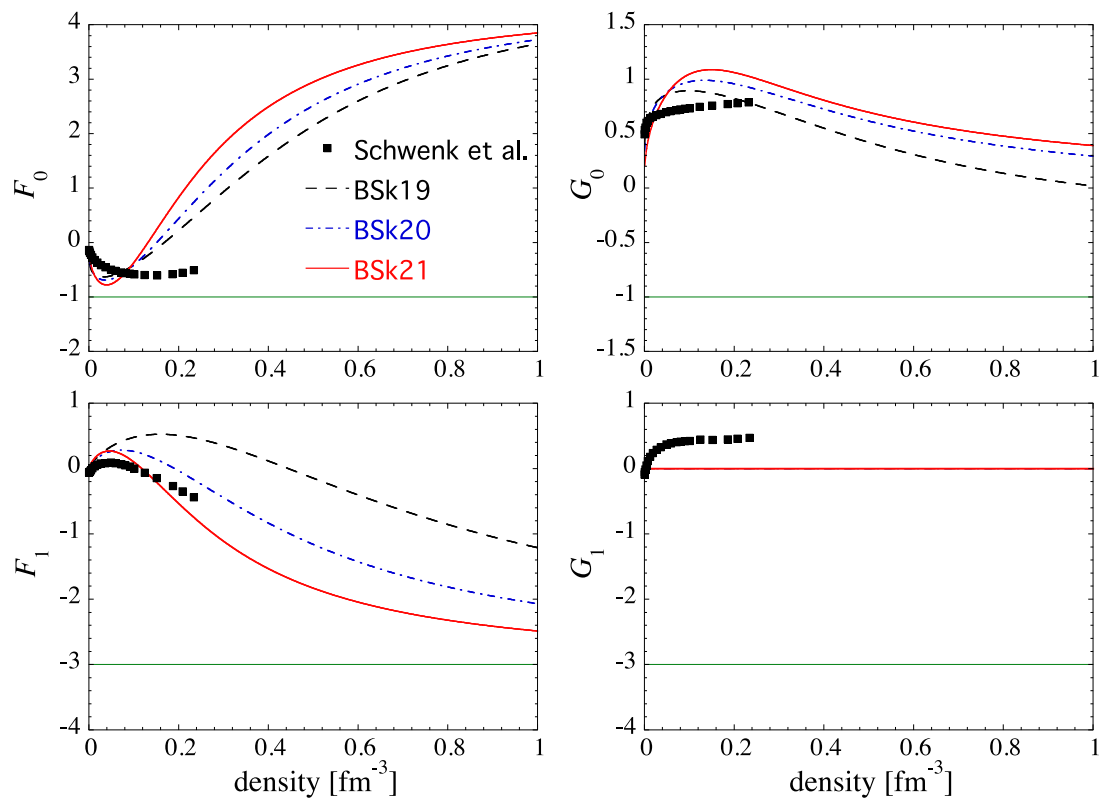

FIG. 12: (Color online) Landau parameters in neutron matter for forces BSk19 - BSk21 (note that the curves for the three forces are indistinguishable from each other in the case of $G_{1}$ ). For comparison we show also the results of BHF calculations from Ref. [72]. We also indicate the stability limit $-(2 \ell+1)$.

\section{NEUTRON-STAR MATTER}

We treat neutron-star matter $\left(\mathrm{N}^{*} \mathrm{M}\right)$, the matter constituting the homogeneous core of neutron stars, as an electrically neutral $\beta$-equilibrated mixture of neutrons, protons, electrons and negative muons (muons were neglected in our article on mass model HFB-18 [4]). Other particles, such as hyperons and quarks, may appear toward the center of the star, but we neglect them here, since the many uncertainties associated with them would serve only to obscure the points that we want to make.

Equation of state and composition. Figure 13 shows the zero-temperature EOS of $\mathrm{N}^{*} \mathrm{M}$ for each of our three new forces (the EOS for the older force, BSk18, is quite similar to that of BSk19). In each case we have, as mentioned earlier, checked that $\mathrm{N}^{*} \mathrm{M}$ remains unpolarized over the whole density range. In Figure 14 we see as a function of density the fraction $Y_{p}$ of nucleons that are protons, while Figure 15 shows the fraction $X_{\mu}$ of leptons that are muons (the number of leptons per nucleon is just $Y_{p}$ ).

Comparing Figure 1 with Figure [13, we see that although the EOS of $\mathrm{N}^{*} \mathrm{M}$ might be considerably softer than the corresponding EOS of NeuM, there is still a correlation between 


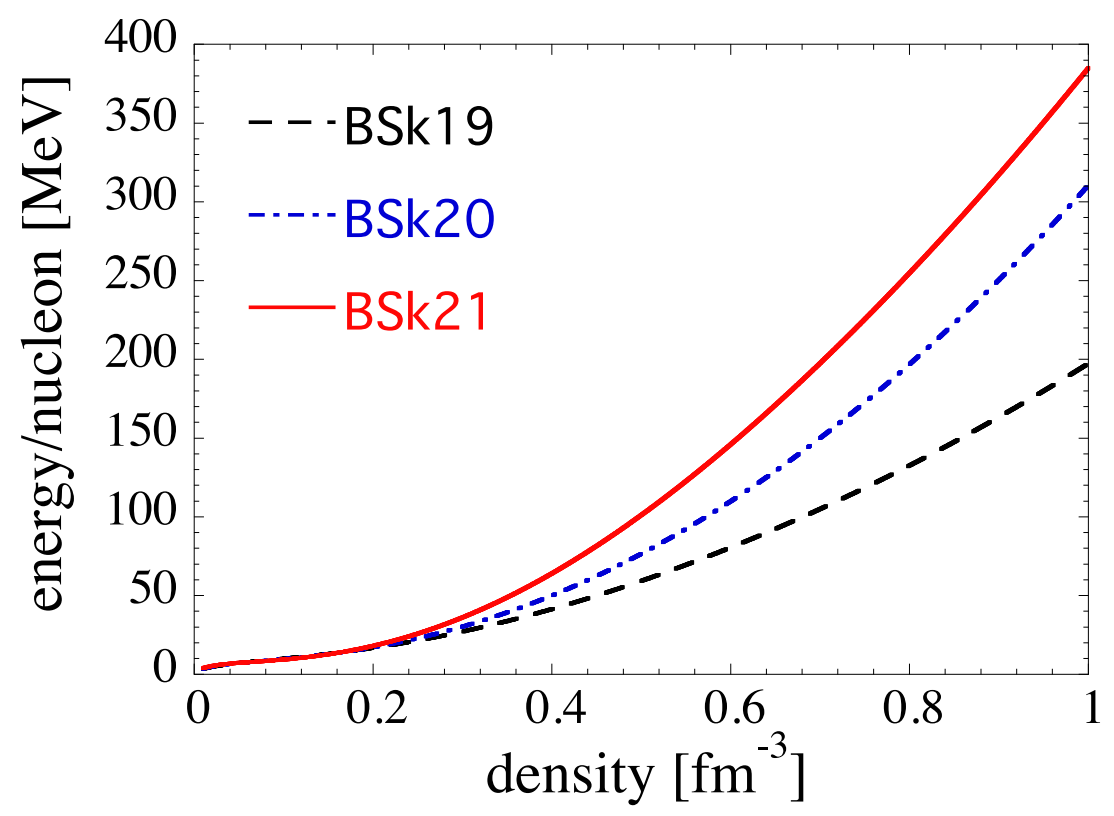

FIG. 13: (Color online) EOSs of neutron-star matter for forces BSk19 - BSk21.

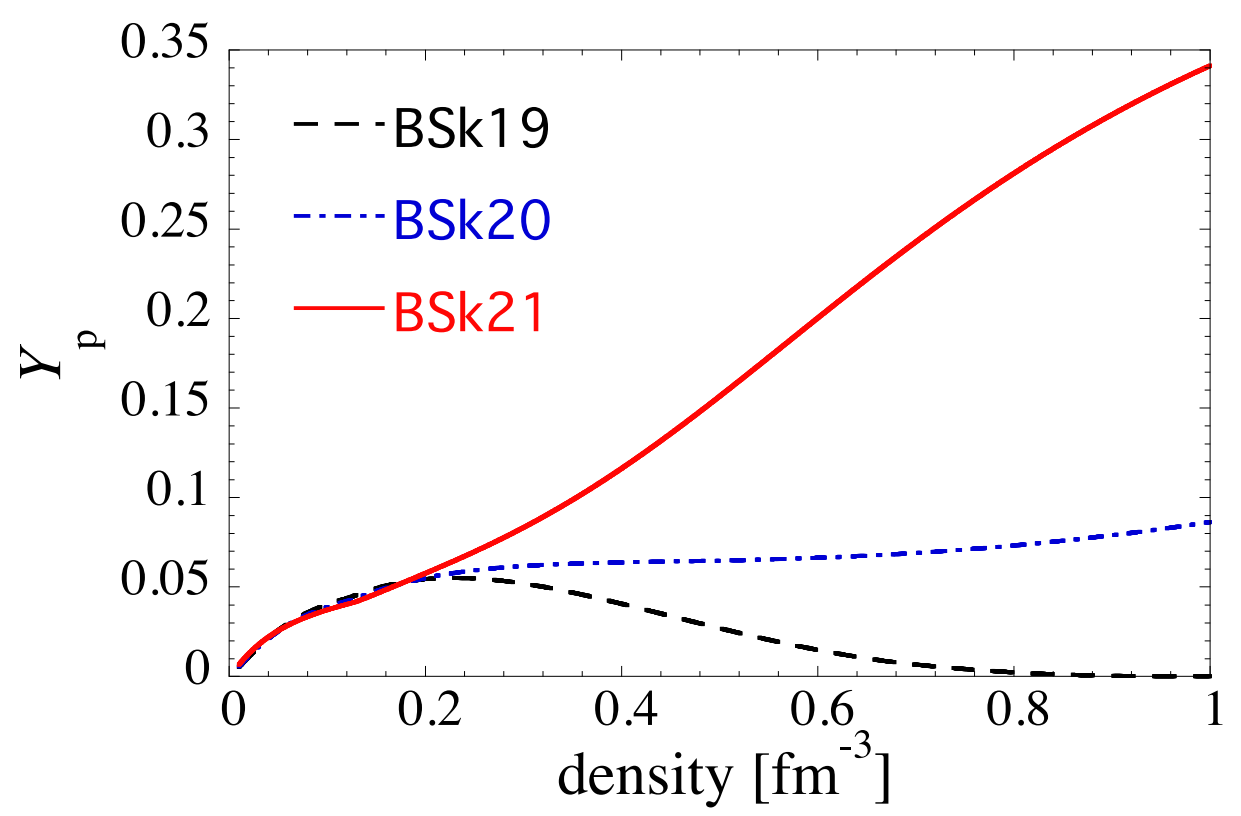

FIG. 14: (Color online) Proton fraction $Y_{p}$ in neutron-star matter for forces BSk19 - BSk21.

the two systems in the sense that the stiffer the EOS of NeuM, the stiffer the EOS of $\mathrm{N}^{*} \mathrm{M}$. Likewise, comparison of Figure 9 with Figure 14 shows that the symmetry energy $e_{s y m}^{(2)}$ is strongly correlated with the protonic fraction $Y_{p}$, as is well known (see, for example, Sec 5.11 .2 of Ref. [73]). It is particularly to be noted that even though the EOS of BSk19 is "supersoft", in the sense that the symmetry energy turns negative at densities encountered 


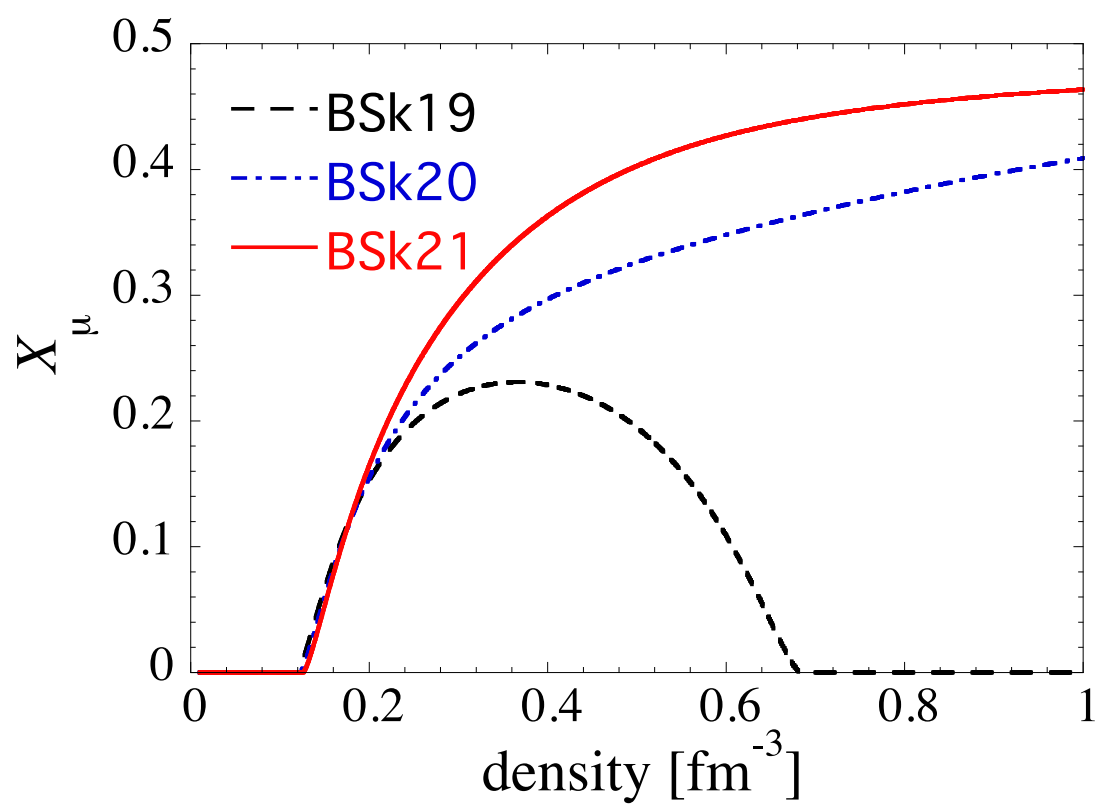

FIG. 15: (Color online) Number of muons per proton in neutron-star matter for forces BSk19 BSk21

in neutron-star cores, it will still support a stable neutron star (contrary to the suggestion of Ref. [74]), since, as seen in Figure [13, the energy per nucleon increases monotonically with density, i.e., the pressure is always positive. Indeed, precisely because the EOS is "supersoft", $\mathrm{N}^{*} \mathrm{M}$ becomes, for BSk19, pure NeuM at higher densities, and the symmetry energy plays no direct role.

The value of $Y_{p}$ found at any given density in a neutron star is of considerable interest, since if, but only if, it exceeds $\approx 11-15 \%$ a direct Urca process of neutrino cooling [75] will be possible. Inspection of Figure 14 tells us that with BSk19 a direct Urca process will be quite impossible, with BSk20 it will be unlikely, while it will certainly occur for BSk21. We recall that the one available experimental result on the high-density behavior of the symmetry energy tends to favor the BSk19 force, thereby casting doubt on the possibility of a direct Urca process. On the other hand, the low luminosity from the pulsar in CTA 1 and from several young supernova remnants likely to contain a still unobserved neutron star [76, 77] might be an indication that a direct Urca process was actually occurring [78, 79]. Evidently, an enormous amount of work, both theoretical and experimental, remains to be done on the question of symmetry energy at the high densities found toward the center of neutron stars. In the meantime, a clear demonstration that a direct Urca process was or was 
not contributing to neutron-star cooling would serve as a most valuable signpost to nuclear physicists.

Causality. A necessary condition for the validity of our forces is that the speed of sound $v_{s}$ in $\mathrm{N}^{*} \mathrm{M}$ must not exceed the speed of light $c$ at the densities encountered in neutron stars. We have therefore calculated the density $\rho_{c}$ above which this condition is violated. The velocity of sound is given by (see Sec 5.13 .3 of Ref. [73])

$$
\frac{v_{s}}{c}=\sqrt{\left(\frac{\mathrm{d} P}{\mathrm{~d} \mathcal{E}}\right)_{\mathrm{fr}}}=\sqrt{\frac{\gamma_{\mathrm{fr}} P}{\mathcal{E}+P}},
$$

in which $P$ is the pressure, $\mathcal{E}$ is the total energy density (including the rest-mass energy) and $\gamma_{\mathrm{fr}}$ is the adiabatic index, defined by

$$
\gamma_{\mathrm{fr}}=\frac{\rho}{P}\left(\frac{\mathrm{d} P}{\mathrm{~d} \rho}\right)_{\mathrm{fr}} ;
$$

the subscript "fr" is to indicate that the derivatives have to evaluated with the composition frozen. The value of $\rho_{c}$ for the different forces are given in Table IV. Even though the contribution of electrons and muons to the EOS is rather small, it was included in $v_{s}$, treating them as relativistic Fermi gases. As can be seen from Figure 13, the stiffer the EOS, the lower $\rho_{c}$ is. For comparison we have also shown in Table IV the critical density $\rho_{c}$ in pure NeuM. For force BSk19 the critical densities in NeuM and in $\mathrm{N}^{*} \mathrm{M}$ are the same because at high densities $\mathrm{N}^{*} \mathrm{M}$ consists of neutrons only. On the other hand, for BSk21 $\mathrm{N}^{*} \mathrm{M}$ contains a sizable amount of proton-lepton pairs, their effect on the EOS and $v_{s}$ being to significantly increase $\rho_{c}$. For all our forces the relatively low values of $\rho_{c}\left(\mathrm{~N}^{*} \mathrm{M}\right)$ that we find may lead to a violation of causality in heavier neutron stars, but in this respect we are limited by the corresponding violation that occurs in the realistic EOSs of NeuM to which we have fitted our forces.

\section{CONCLUSIONS}

This article describes the latest effort in our long-standing quest for effective forces (Skyrme plus contact pairing) that will lead to high-precision mass models, while at the same time respecting the physical constraints appropriate to the neutron-rich environments found in neutron stars and supernova cores. Specifically, we present here a family of three generalized Skyrme forces, each of which, when taken with the appropriate pairing force, 
leads to a mass model that fits essentially all the mass data [2] with an rms deviation of about $0.58 \mathrm{MeV}$. However, although these models give very similar extrapolations out to the neutron drip line, the corresponding Skyrme forces are distinguished by the very different way in which the symmetry energy of each varies at the high densities found in the core of neutron stars, ranging from the supersoft (i.e., negative symmetry energy) to the very stiff. This degree of flexibility, maintaining a high quality mass fit with very different neutronmatter constraints, has been made possible only through the introduction of the $t_{4}$ and $t_{5}$ terms, i.e., density-dependent generalizations of the usual $t_{1}$ and $t_{2}$ terms, respectively. Despite these differences, each of these Skyrme forces finds theoretical support in the sense of having been fitted to one realistic many-body calculation or another of neutron matter. Moreover, the neutron matter corresponding to each of these forces is stable against unphysical transitions, such as a ferromagnetic flip. Finally, in symmetric nuclear matter the distribution of potential energy among the different spin-isospin channels is in qualitative accord with realistic many-body calculations.

We claim now that with the forces presented here, along with their respective mass models, it has become possible for the first time to adopt a unified treatment at the level of effective forces of all the nuclear properties of the highly neutron-rich systems of astrophysical interest. Thus by constraining the forces to fit not only all the available mass data but also the EOS of neutron matter our HFB mass models may be expected to provide the most reliable estimates of the masses of the experimentally inaccessible highly neutron-rich nuclei that appear in the outer crust of neutron stars and that are involved in the $r$-process (note that the predictions of our three forces for neutron matter begin to diverge from each other only at densities of about $\left.2 \rho_{0}\right)$. Moreover, since our forces, having been fitted not only to neutron matter but also to nuclear masses, take account of both inhomogeneities and the presence of protons they can be used with confidence to calculate the EOS of the inner crust of neutron stars. Indeed, since our forces do not lead to any unphysical instability in neutron matter they can likewise be used for the study of the core of neutron stars, whence a unified treatment of the whole star becomes possible.

Of course, the three different Skyrme forces will make quite different predictions for the properties of the core (and thus for global properties such as the mass - radius relationship), but this simply reflects our present ignorance of the properties of neutron matter at high density. But even if many-body theory could be developed to the point where the EOS 
of neutron matter could be tied down unambiguously, there would still be uncertainties associated with the possible presence of hyperons and other exotica. Thus we believe that the ultimate discrimination among our different forces will come either from experiment or observation of neutron stars. (As for experiment, we stress that measurement must relate to high densities: we cannot draw conclusions about the high-density behavior from low-density quantities such as the symmetry-compressibility coefficients $K_{\text {sym }}$ or $K_{\tau}$, even if measured with sufficient precision.) But whichever of our three forces is ultimately favored, we have shown here that the Skyrme form of effective interaction is flexible enough to permit a description of the nuclear physics of the entire neutron star with a unique set of parameters.

We also want to be able to apply the same Skyrme force to the calculation of nuclear quantities relevant to the $r$-process. In addition to masses, such quantities include beta strength functions, fission barriers and level densities. In connection with the first of these quantities an outstanding difficulty with all our previously published HFB models has been that of obtaining a reasonable value for the Landau parameter $G_{0}^{\prime}$; for the first time we can claim that with our forces BSk19 - BSk21 we have resolved this problem. Previous articles have already dealt successfully with fission barriers [15] and level densities [80, 81].

\section{Acknowledgments}

We thank M. Baldo, U. Garg and U. Lombardo for valuable exchanges. This work was financially supported by the FNRS (Belgium), the Communauté française de Belgique (Actions de Recherche Concertées), CompStar (a Research Networking Programme of the European Science Foundation) and the NSERC (Canada). J. M. P. is grateful to Bureau des relations internationales of the Université Libre de Bruxelles for financial support for the month of November 2009.

\section{Appendix A: Microscopic calculation of collective quadrupole energy}

Systematic calculations of the collective quadrupole energy have recently been performed on the basis of the D1M Gogny interaction by solving the collective Schrödinger equation with the five-dimensional collective Hamiltonian [26]. The five collective quadrupole coordinates correspond to the rotation, the quadrupole vibration and the coupling between 


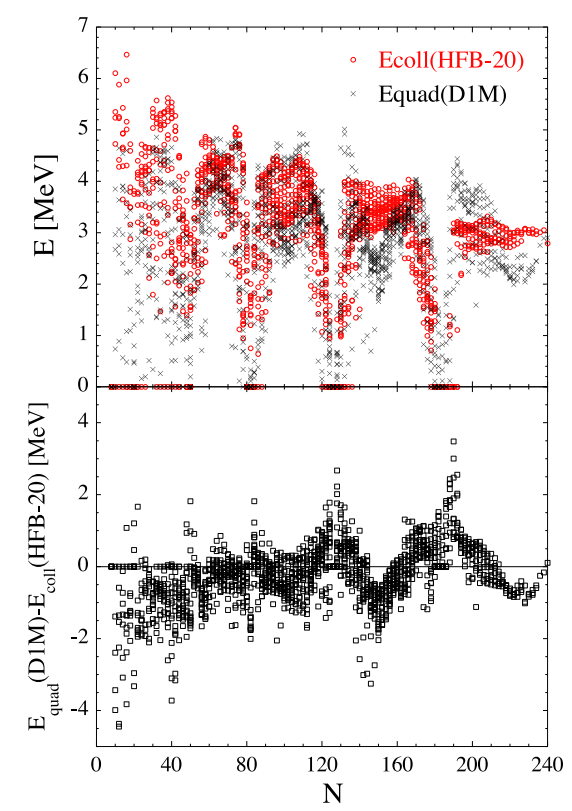

FIG. 16: (Color online) (Upper panel) Comparison between the D1M quadrupole correction energy (crosses) [26] and the HFB-20 collective energy (circles) as a function of $N$ for the 1770 even-even nuclei with $Z, N>8$ and $Z \leq 110$. (Lower panel) Differences between the D1M quadrupole correction energy and the HFB-19 collective energy.

these collective modes; it includes axial as well as triaxial quadrupole deformations. The corresponding quadrupole correction energy obtained with the D1M force is compared in Figure 16 with the collective correction energy (6) for the 1770 even-even nuclei with $Z, N>8$ and $Z \leq 110$. Globally, our simple approximation is seen to be in relatively good agreement with the D1M quadrupole energies, so we can be confident that most of the quadrupole effects are indeed explicitly treated and not taken at the level of the mean field calculation (hence not absorbed into the effective force). Some systematic deviations between both approaches can be observed in the lower panel of Figure 16. Interestingly these differences are correlated to those found between experimental and D1M masses (see in particular Figure 1 of Ref. [26]) for $N>60$ nuclei, so mid-shell correlations, in particular around $N \simeq 100$ and 150, may in fact be underestimated by the collective Hamiltonian model. 


\section{Appendix B: Landau parameters in neutron matter}

The expressions of the Landau parameters for symmetric nuclear matter associated with generalized Skyrme forces (2) were given in Ref. [4]. Here we provide the expressions of the Landau parameters in pure neutron matter, using the same notations.

$$
\begin{gathered}
F_{0}^{\mathrm{NeuM}}=N\left[2 C_{0}^{\rho}+2 C_{1}^{\rho}+2 k_{\mathrm{F}}^{2}\left(C_{0}^{\tau}+C_{1}^{\tau}\right)+4 \rho\left(\frac{\mathrm{d} C_{0}^{\rho}}{\mathrm{d} \rho}+\frac{\mathrm{d} C_{1}^{\rho}}{\mathrm{d} \rho}\right)\right. \\
\left.+\rho^{2}\left(\frac{\mathrm{d}^{2} C_{0}^{\rho}}{\mathrm{d} \rho^{2}}+\frac{\mathrm{d}^{2} C_{1}^{\rho}}{\mathrm{d} \rho^{2}}\right)+\rho \tau\left(\frac{\mathrm{d}^{2} C_{0}^{\tau}}{\mathrm{d} \rho^{2}}+\frac{\mathrm{d}^{2} C_{1}^{\tau}}{\mathrm{d} \rho^{2}}\right)+\left(\frac{\mathrm{d} C_{0}^{\tau}}{\mathrm{d} \rho}+\frac{\mathrm{d} C_{1}^{\tau}}{\mathrm{d} \rho}\right)\left(2 \tau+2 \rho k_{\mathrm{F}}^{2}\right)\right] \\
F_{1}^{\mathrm{NeuM}}=-2 N\left(C_{0}^{\tau}+C_{1}^{\tau}\right) k_{\mathrm{F}}^{2} \\
G_{0}^{\text {NeuM }}=2 N\left[C_{0}^{s}+C_{1}^{s}+k_{\mathrm{F}}^{2}\left(C_{0}^{T}+C_{1}^{T}\right)\right]
\end{gathered}
$$

and

$$
G_{1}^{\mathrm{NeuM}}=-2 N k_{\mathrm{F}}^{2}\left(C_{0}^{T}+C_{1}^{T}\right)
$$

where $N$ is the density of s.p. states at the Fermi level

$$
N=\frac{M_{n}^{*} k_{\mathrm{F}}}{\hbar^{2} \pi^{2}}
$$

$k_{\mathrm{F}}=\left(3 \pi^{2} \rho\right)^{1 / 3}$ and $M_{n}^{*}$ is the neutron effective mass given by

$$
\frac{M_{n}}{M_{n}^{*}}=1+\frac{2 M_{n}}{\hbar^{2}}\left(C_{0}^{\tau}+C_{1}^{\tau}\right) \rho .
$$

[1] S. Goriely, N. Chamel, and J. M. Pearson, Phys. Rev. Lett. 102, 152503 (2009).

[2] G. Audi, A.H. Wapstra, and C. Thibault, Nucl. Phys. A729, 337 (2003).

[3] M. Onsi, A. K. Dutta, H. Chatri, S. Goriely, N. Chamel, and J. M. Pearson, Phys. Rev. C 77 065805 (2008).

[4] N. Chamel, S. Goriely, and J. M. Pearson, Phys. Rev. C 80, 065804 (2009).

[5] B. Friedman and V. R. Pandharipande, Nucl. Phys. A361, 502 (1981). 
[6] A. Akmal, V. R. Pandharipande, and D. G. Ravenhall, Phys. Rev. C 58, 1804 (1998).

[7] Z. Xiao, B.-A. Li, L.-W. Chen, G.-C. Yong, and M. Zhang, Phys. Rev. Lett. 102, 062502 (2009).

[8] Z. H. Li and H.-J. Schulze, Phys. Rev. C 78, 028801 (2008).

[9] S. Gandolfi, A. Yu. Illarionov, K. E. Schmidt, F. Pederiva, and S. Fantoni, Phys. Rev. C 79, 054005 (2009).

[10] L .G. Cao, U. Lombardo, and P. Schuck, Phys. Rev. C 74, 064301 (2006).

[11] N. Chamel, S. Goriely, and J. M. Pearson, Nucl. Phys. A812, 72 (2008).

[12] S. Goriely, M. Samyn, P.-H. Heenen, J. M. Pearson, and F. Tondeur, Phys. Rev. C 66, 024326 (2002).

[13] S. Goriely, M. Samyn, J. M. Pearson, and M. Onsi, Nucl. Phys. A750, 425 (2005).

[14] M. Bender, P.-H. Heenen, and P. Bonche, Phys. Rev. C 70, 054304 (2004).

[15] S. Goriely, M. Samyn, and J.M. Pearson, Phys. Rev C 75, 064312 (2007).

[16] S. Goriely and J. M. Pearson, Phys. Rev. C 77, 031301(R) (2008).

[17] J. Dobaczewski and J. Dudek, Phys. Rev. C 52, 1827 (1995).

[18] M. Bender, J. Dobaczewski, J. Engel, and W. Nazarewicz, Phys. Rev. C 65, 054322 (2002).

[19] S. Perez-Martin and L. M. Robledo, Phys. Rev. C 78, 014304 (2008).

[20] S. Goriely, M. Samyn, M. Bender, and J. M. Pearson, Phys. Rev. C 68, 054325 (2003).

[21] G. Colò, N. Van Giai, J. Meyer, K. Bennaceur, and P. Bonche, Phys. Rev. C 70, 024307 (2004).

[22] R.B. Wiringa, V. Fiks, and A. Fabrocini, Phys. Rev. C 38, 1010 (1988).

[23] M. Baldo, P. Schuck, and X. Viñas, Phys. Lett. B663, 390 (2008).

[24] P. Möller, J. R. Nix, W.D. Myers, and W.J. Swiatecki, At. Data Nucl. Data Tables 59, 185 (1995).

[25] I. Angeli, At. Data and Nucl. Data Tables 87, 185 (2004).

[26] S. Goriely, S. Hilaire, M. Girod, and S. Péru, Phys. Rev. Lett. 102, 242501 (2009).

[27] B. L. Berman and S. C. Fultz, Rev. Mod. Phys. 47, 713 (1975).

[28] T. Lesinski, K. Bennaceur, T. Duguet, and J. Meyer, Phys. Rev. C 74, 044315 (2006).

[29] L.G. Cao, U. Lombardo, C.W. Shen, and Nguyen Van Giai, Phys. Rev. C 73, 014313 (2006).

[30] D. Vautherin and D. M. Brink, Phys. Rev. C 5, 626 (1972).

[31] M. Farine, J. M. Pearson, and F. Tondeur, Nucl. Phys. A696, 396 (2001). 
[32] X.R. Zhou, G.F. Burgio, U. Lombardo, H.-J. Schulze and W. Zuo, Phys. Rev C 69, 018801 (2004).

[33] U.Lombardo, private communication.

[34] M. Baldo, private communication.

[35] A. W. Steiner, Phys. Rev. C 74, 045808 (2006).

[36] P. Danielewicz, R. Lacey, and W. G. Lynch, Science 298, 1592 (2002).

[37] P. G. Krastev and F. Sammarruca, Phys. Rev. C 74, 025808 (2006).

[38] M. Farine, J. M. Pearson, and B. Rouben, Nucl. Phys. A304, 317 (1978).

[39] F. Tondeur, M. Brack, M. Farine, and J. M. Pearson, Nucl. Phys. A420, 297 (1984).

[40] P.-G. Reinhard, Nucl. Phys. A649, 305c (1999).

[41] M. Warda, X.Viñas, X. Roca-Maza, and M. Centelles, Phys. Rev. C 80, 024316 (2009).

[42] P.-G. Reinhard and W. Nazarewicz, arXiv:1002.4140

[43] M. B. Tsang, Y. Zhang, P. Danielewicz, M. Famiano, Z. Li, W. G. Lynch, and A. W. Steiner, Phys. Rev. Lett. 102, 122701 (2009).

[44] L.-W. Chen, C. M. Ko, and B.-A. Li, Phys. Rev. C 72, 064309 (2005).

[45] M. A. Famiano, T. Liu, W. G. Lynch, M. Mocko, A. M. Rogers, M. B. Tsang, M. S. Wallace, R. J. Charity, S. Komarov, D. G. Sarantites, L. G. Sobotka, and G. Verde, Phys. Rev. Lett. 97, 052701 (2006).

[46] B. A. Brown, Phys. Rev. Lett. 85, 5296 (2000).

[47] S. Typel and B. A. Brown, Phys. Rev. C 64, 027302 (2001).

[48] W. D. Myers and W. J. Swiatecki, Ann. Phys. (NY), 55, 395 (1969).

[49] M. Farine, J. Côté, and J. M. Pearson, Nucl. Phys. A338, 86 (1980).

[50] M. Farine, J. Côté, and J. M. Pearson, Phys. Rev. C 24, 303 (1981).

[51] J. Jastrzebski, A. Trzcinska, P. Lubinski, B. Klos, F. J. Hartmann, T. von Egidy, and S. Wycech, Int. J. Mod. Phys. E 13, 343 (2004).

[52] G. W. Hoffmann, L. Ray, M. Barlett, J. McGill, G. S. Adams, G. J. Igo, F. Irom, A. T. M. Wang, C. A. Whitten,Jr., R. L. Boudrie, J. F. Amann, C. Glashausser, N. M. Hintz, G. S. Kyle, and G. S. Blanpied, Phys. Rev. C 21, 1488 (1980).

[53] B. A. Brown, G. Shen, G. C. Hillhouse, J. Meng, and A. Trzcińska, Phys. Rev. C 76, 034305 (2007).

[54] R. Michaels, P. A. Souder, and G. M. Urciouli, 
http://hallaweb.jlab.org/parity/prex/pbup2005.pdf.

[55] I. Vidaña, C. Providência, A. Polls, and A. Rios, Phys. Rev. C 80, 045806 (2009).

[56] R. Machleidt, Adv. Nucl. Phys. 19, 189 (1989).

[57] L.-W. Chen, B.-J. Cai, C. M. Ko, B.-A. Li, C. Shen, and J. Xu, Phys. Rev. C 80, 014322 (2009).

[58] T. Li, U. Garg, Y. Liu, R. Marks, B. K. Nayak, P. V. Madhusudhana Rao, M. Fujiwara, H. Hashimoto, K. Nakanishi, S. Okomura, M. Yosoi, M. Ichikawa, M. Itoh, R. Matsuo, T. Terazono, M. Uchida, Y. Iwao, T. Kawabata, T. Murakami, H. Sakaguchi, S. Terashima, Y. Yasuda, J. Zenihiro, H. Akimune, K. Kawase, and M. N. Harakeh, Phys. Rev. C 81, 034309 (2010).

[59] J.P. Blaizot, Phys. Rep. 64, 171 (1980).

[60] J. Piekarewicz and M. Centelles, Phys. Rev. C79, 054311 (2009).

[61] J. Piekarewicz, J. Phys. G 37, 064038 (2010).

[62] J. M. Pearson, N. Chamel, and S. Goriely, Phys. Rev. C 82, 037301 (2010).

[63] G. Colò, Bulk Nuclear Properties, Conference Proceedings (AIP, Melville, NY, 2009), Vol. 1128 , p. 59.

[64] B. K. Jennings and A. D. Jackson, Phys. Rep. 66, 141 (1980).

[65] J.P. Blaizot, J.F. Berger, J. Dechargé, and M. Girod, Nucl. Phys. A591, 435 (1995).

[66] J. Piekarewicz, Phys. Rev. C 76, 031301(R) (2007).

[67] B. G. Todd-Rutel and J. Piekarewicz, Phys. Rev. Lett. 95, 122501 (2005).

[68] W. Zuo, C. Shen, U. Lombardo, Phys. Rev. C 67, 037301 (2003).

[69] I.N. Borzov, S.V. Tolokonnikov and S. A. Fayans, Sov. J. Nucl. Phys. 40, 732 (1984).

[70] B.L. Friman, and A.K. Dhar, Phys. Lett. B 85, 1 (1979).

[71] C. Shen, U. Lombardo, N. Van Giai, and W. Zuo, Phys. Rev. C 68, 055802 (2003).

[72] A. Schwenk, B. Friman, and G. E. Brown, Nucl. Phys. A713, 191 (2003).

[73] P. Haensel, A.Y. Potekhin, and D.G. Yakovlev, Neutron stars 1: equation of state and structure (Springer, Berlin, 2007).

[74] D.-H. Wen, B.-A. Li, and L.-W. Chen, Phys. Rev. Lett. 103, 211102 (2009).

[75] J. M. Lattimer, C. J. Pethick, M. Prakash, and P. Haensel, Phys. Rev. Lett. 66, 2701 (1991).

[76] D.L. Kaplan, D. A. Frail, B.M. Gaensler, E. V. Gotthelf, S.R. Kulkarni, P.O. Slane, and A. Nechita, Astrophys. J. Suppl. Series 153, 269 (2004). 
[77] D.L. Kaplan, B.M. Gaensler, S.R. Kulkarni, and P.O. Slane, Astrophys. J. Suppl. Series 163, 344 (2006).

[78] P.S. Shternin and D.G. Yakovlev, Astron. Lett. 34,675 (2008).

[79] D. Page, J. M. Lattimer, M. Prakash, and A. W. Steiner, Astrophys. J. 707, 1131 (2009).

[80] S. Goriely, M. Samyn, and J.M. Pearson, Nucl. Phys. A773, 279 (2006).

[81] S. Hilaire, and S. Goriely, Nucl. Phys. A779 63 (2006). 
TABLE I: Forces BSk19 - BSk21: lines 1-16 show the Skyrme parameters, lines 17-21 the pairing parameters, and the last 4 lines the Wigner parameters (see text for further details). Note that it is more convenient to show the $x_{2}$ parameter in the form $t_{2} x_{2}$, the only combination in which $x_{2}$ enters into the formalism. For convenience of comparison we also show the force BSk18 [4].

\begin{tabular}{|c|c|c|c|c|}
\hline & BSk19 & BSk20 & $\mathrm{BSk} 21$ & BSk18 \\
\hline$t_{0}\left[\mathrm{MeV} \mathrm{fm}{ }^{3}\right]$ & -4115.21 & -4056.04 & -3961.39 & -1837.96 \\
\hline$t_{1}\left[\mathrm{MeV} \mathrm{fm}^{5}\right]$ & 403.072 & 438.219 & 396.131 & 428.880 \\
\hline$t_{2}\left[\mathrm{MeV} \mathrm{fm}^{5}\right]$ & 0 & 0 & 0 & -3.23704 \\
\hline$t_{3}\left[\mathrm{MeV} \mathrm{fm}{ }^{3+3 \alpha}\right]$ & 23670.4 & 23256.6 & 22588.2 & 11528.9 \\
\hline$t_{4}\left[\mathrm{MeV} \mathrm{fm}^{5+3 \beta}\right]$ & -60.0 & -100.000 & -100.000 & -400.000 \\
\hline$t_{5}\left[\mathrm{MeV} \mathrm{fm}{ }^{5+3 \gamma}\right]$ & -90.0 & -120.000 & -150.000 & -400.000 \\
\hline$x_{0}$ & 0.398848 & 0.569613 & 0.885231 & 0.421290 \\
\hline$x_{1}$ & -0.137960 & -0.392047 & 0.0648452 & -0.907175 \\
\hline$t_{2} x_{2}\left[\mathrm{MeV} \mathrm{fm}{ }^{5}\right]$ & -1055.55 & -1147.64 & -1390.38 & -186.837 \\
\hline$x_{3}$ & 0.375201 & 0.614276 & 1.03928 & 0.683926 \\
\hline$x_{4}$ & -6.0 & -3.00000 & 2.00000 & -2.00000 \\
\hline$x_{5}$ & -13.0 & -11.0000 & -11.0000 & -2.00000 \\
\hline$W_{0}\left[\mathrm{MeV} \mathrm{fm} \mathrm{fm}^{5}\right]$ & 110.802 & 110.228 & 109.622 & 138.904 \\
\hline$\alpha$ & $1 / 12$ & $1 / 12$ & $1 / 12$ & 0.3 \\
\hline$\beta$ & $1 / 3$ & $1 / 6$ & $1 / 2$ & 1.0 \\
\hline$\gamma$ & $1 / 12$ & $1 / 12$ & $1 / 12$ & 1.0 \\
\hline$f_{n}^{+}$ & 1.00 & 1.00 & 1.00 & 1.00 \\
\hline$f_{n}^{-}$ & 1.05 & 1.06 & 1.05 & 1.06 \\
\hline$f_{p}^{+}$ & 1.10 & 1.09 & 1.07 & 1.04 \\
\hline$f_{p}^{-}$ & 1.17 & 1.16 & 1.13 & 1.09 \\
\hline$\varepsilon_{\Lambda}[\mathrm{MeV}]$ & 16.0 & 16.0 & 16.0 & 16.0 \\
\hline$V_{W}[\mathrm{MeV}]$ & -2.00 & -2.10 & -1.80 & -2.10 \\
\hline$\lambda$ & 250 & 280 & 280 & 340 \\
\hline$V_{W}^{\prime}[\mathrm{MeV}]$ & 1.16 & 0.96 & 0.96 & 0.74 \\
\hline$A_{0}$ & 24 & 24 & 24 & 28 \\
\hline
\end{tabular}


TABLE II: Parameters of Eq.(66) for collective correction to models HFB-19, HFB-20 and HFB-21.

\begin{tabular}{|c|c|}
\hline$b(\mathrm{MeV})$ & 0.80 \\
$c$ & 10 \\
$d(\mathrm{MeV})$ & 3.4 \\
$l$ & 17 \\
$\beta_{2}^{0}$ & 0.1 \\
\hline
\end{tabular}

TABLE III: Rms $(\sigma)$ and mean $(\bar{\epsilon})$ deviations between data and predictions for models HFB-19, HFB-20 and HFB-21; for convenience of comparison we also show model HFB-18 [4]. The first pair of lines refers to all the 2149 measured masses $M$ that were fitted [2], the second pair to the masses $M_{n r}$ of the subset of 185 neutron-rich nuclei with $S_{n} \leq 5.0 \mathrm{MeV}$, the third pair to the neutron separation energies $S_{n}$ (1988 measured values), the fourth pair to beta-decay energies $Q_{\beta}$ (1868 measured values) and the fifth pair to charge radii (782 measured values [25]). The last line shows the calculated neutron-skin thickness of ${ }^{208} \mathrm{~Pb}$ for these models.

\begin{tabular}{|c|ccc|c|}
\hline & HFB-19 & HFB-20 & HFB-21 & HFB-18 \\
\hline$\sigma(M)[\mathrm{MeV}]$ & 0.583 & 0.583 & 0.577 & 0.585 \\
$\bar{\epsilon}(M)[\mathrm{MeV}]$ & -0.038 & 0.021 & -0.054 & 0.007 \\
$\sigma\left(M_{n r}\right)[\mathrm{MeV}]$ & 0.803 & 0.790 & 0.762 & 0.758 \\
$\bar{\epsilon}\left(M_{n r}\right)[\mathrm{MeV}]$ & 0.243 & 0.217 & -0.086 & 0.172 \\
$\sigma\left(S_{n}\right)[\mathrm{MeV}]$ & 0.502 & 0.525 & 0.532 & 0.487 \\
$\bar{\epsilon}\left(S_{n}\right)[\mathrm{MeV}]$ & -0.015 & -0.012 & -0.009 & -0.012 \\
$\sigma\left(Q_{\beta}\right)[\mathrm{MeV}]$ & 0.612 & 0.620 & 0.620 & 0.561 \\
$\bar{\epsilon}\left(Q_{\beta}\right)[\mathrm{MeV}]$ & 0.027 & 0.024 & 0.000 & 0.025 \\
$\sigma\left(R_{c}\right)[\mathrm{fm}]$ & 0.0283 & 0.0274 & 0.0270 & 0.0274 \\
$\bar{\epsilon}\left(R_{c}\right)[\mathrm{fm}]$ & -0.0032 & 0.0009 & -0.0014 & 0.0016 \\
$\theta\left({ }^{208} \mathrm{~Pb}\right)[\mathrm{fm}]$ & 0.140 & 0.140 & 0.137 & 0.150 \\
\hline
\end{tabular}


TABLE IV: Parameters of infinite nuclear matter for forces BSk19, BSk20 and BSk21; for convenience of comparison we also show force BSk18 [4].

\begin{tabular}{|c|ccc|c|}
\hline & BSk19 & BSk20 & BSk21 & BSk18 \\
\hline$a_{v}[\mathrm{MeV}]$ & -16.078 & -16.080 & -16.053 & -16.063 \\
$\rho_{0}\left[\mathrm{fm}^{-3}\right]$ & 0.1596 & 0.1596 & 0.1582 & 0.1586 \\
$J[\mathrm{MeV}]$ & 30.0 & 30.0 & 30.0 & 30.0 \\
$K_{v}[\mathrm{MeV}]$ & 237.3 & 241.4 & 245.8 & 241.8 \\
$K^{\prime}[\mathrm{MeV}]$ & 297.8 & 282.2 & 274.1 & 363.8 \\
$L_{[\mathrm{MeV}]}$ & 31.9 & 37.4 & 46.6 & 36.2 \\
$K_{s y m}[\mathrm{MeV}]$ & -191.4 & -136.5 & -37.2 & -180.9 \\
$K_{\tau}[\mathrm{MeV}]$ & -342.8 & -317.1 & -264.6 & -343.7 \\
$K_{c o u l}[\mathrm{MeV}]$ & -5.093 & -5.158 & -5.186 & -4.897 \\
$M_{s}^{*} / M$ & 0.80 & 0.80 & 0.80 & 0.80 \\
$M_{v}^{*} / M$ & 0.61 & 0.65 & 0.71 & 0.79 \\
$F_{0}$ & -0.14 & -0.13 & -0.10 & -0.12 \\
$F_{0}^{\prime}$ & 0.96 & 0.96 & 0.97 & 0.97 \\
$F_{1}$ & -0.60 & -0.60 & -0.60 & -0.60 \\
$F_{1}^{\prime}$ & 0.96 & 0.69 & 0.38 & 0.032 \\
$G_{0}$ & -0.016 & 0.25 & 0.56 & -0.33 \\
$G_{0}^{\prime}$ & 0.95 & 0.95 & 0.95 & 0.46 \\
$G_{1}$ & 0 & 0 & 0 & 1.23 \\
$G_{1}^{\prime}$ & 0 & 0 & 0 & 0.50 \\
$\rho_{c}\left(\mathrm{~N}^{*} \mathrm{M}\right)\left[\mathrm{NeuM}{ }^{-3}\right]$ & 1.45 & 0.98 & 0.99 & 1.77 \\
& 1.45 & 0.95 & 0.69 & 1.77 \\
\hline
\end{tabular}


TABLE V: Single-particle neutron levels in ${ }^{208} \mathrm{~Pb}(\mathrm{MeV})$. Experimental values are taken from Ref. [30]. The asterisk denotes the Fermi level. The quantity $\Delta_{n}$ is the interval between the centroids of the $2 f$ and $3 d$ doublets.

\begin{tabular}{|c|cc|c|}
\hline Level & HFB-18 & HFB-20 & Expt. \\
\hline $1 s_{1 / 2}$ & -51.0 & -49.1 & - \\
$\ldots$ & $\ldots$ & $\ldots$ & $\ldots$ \\
$1 h_{9 / 2}$ & -12.6 & -11.7 & -10.9 \\
$2 f_{7 / 2}$ & -11.6 & -11.3 & -9.7 \\
$1 i_{13 / 2}$ & -9.4 & -9.7 & -9.0 \\
$3 p_{3 / 2}$ & -8.8 & -8.7 & -8.3 \\
$2 f_{5 / 2}$ & -8.8 & -8.6 & -8.0 \\
$3 p_{1 / 2} *$ & -7.7 & -7.7 & -7.4 \\
$2 g_{9 / 2}$ & -3.5 & -3.7 & -3.9 \\
$1 i_{11 / 2}$ & -2.7 & -2.3 & -3.2 \\
$1 j_{15 / 2}$ & -1.2 & -1.8 & -2.5 \\
$3 d_{5 / 2}$ & -1.2 & -1.5 & -2.4 \\
$4 s_{1 / 2}$ & -0.7 & -0.9 & -1.9 \\
$2 g_{7 / 2}$ & -0.4 & -0.7 & -1.5 \\
$3 d_{3 / 2}$ & -0.2 & -0.5 & -1.4 \\
\hline$\Delta_{n}$ & 9.6 & 9.0 & 7.0 \\
\hline
\end{tabular}


TABLE VI: Single-particle proton levels in ${ }^{208} \mathrm{~Pb}(\mathrm{MeV})$. Experimental values are taken from Ref. [30]. The asterisk denotes the Fermi level. The quantity $\Delta_{p}$ is the interval between the centroids of the $1 g$ and $2 f$ doublets.

\begin{tabular}{|c|cc|c|}
\hline Level & HFB-18 & HFB-20 & Expt. \\
\hline $1 s_{1 / 2}$ & -39.8 & -41.0 & - \\
$\ldots$ & $\ldots$ & $\ldots$ & $\ldots$ \\
$1 g_{9 / 2}$ & -16.3 & -16.6 & -15.4 \\
$1 g_{7 / 2}$ & -13.0 & -12.7 & -11.4 \\
$2 d_{5 / 2}$ & -10.2 & -10.3 & -9.7 \\
$1 h_{11 / 2}$ & -8.8 & -9.0 & -9.4 \\
$2 d_{3 / 2}$ & -8.4 & -8.6 & -8.4 \\
$3 s_{1 / 2} *$ & -7.7 & -7.8 & -8.0 \\
$1 h_{9 / 2}$ & -4.0 & -3.5 & -3.8 \\
$2 f_{7 / 2}$ & -2.2 & -2.2 & -2.9 \\
$1 i_{13 / 2}$ & -1.0 & -1.1 & -2.2 \\
$3 p_{3 / 2}$ & 1.0 & 0.9 & -1.0 \\
$2 f_{5 / 2}$ & 0.2 & 0.1 & -0.5 \\
\hline$\Delta_{p}$ & 13.6 & 13.7 & 11.7 \\
\hline
\end{tabular}




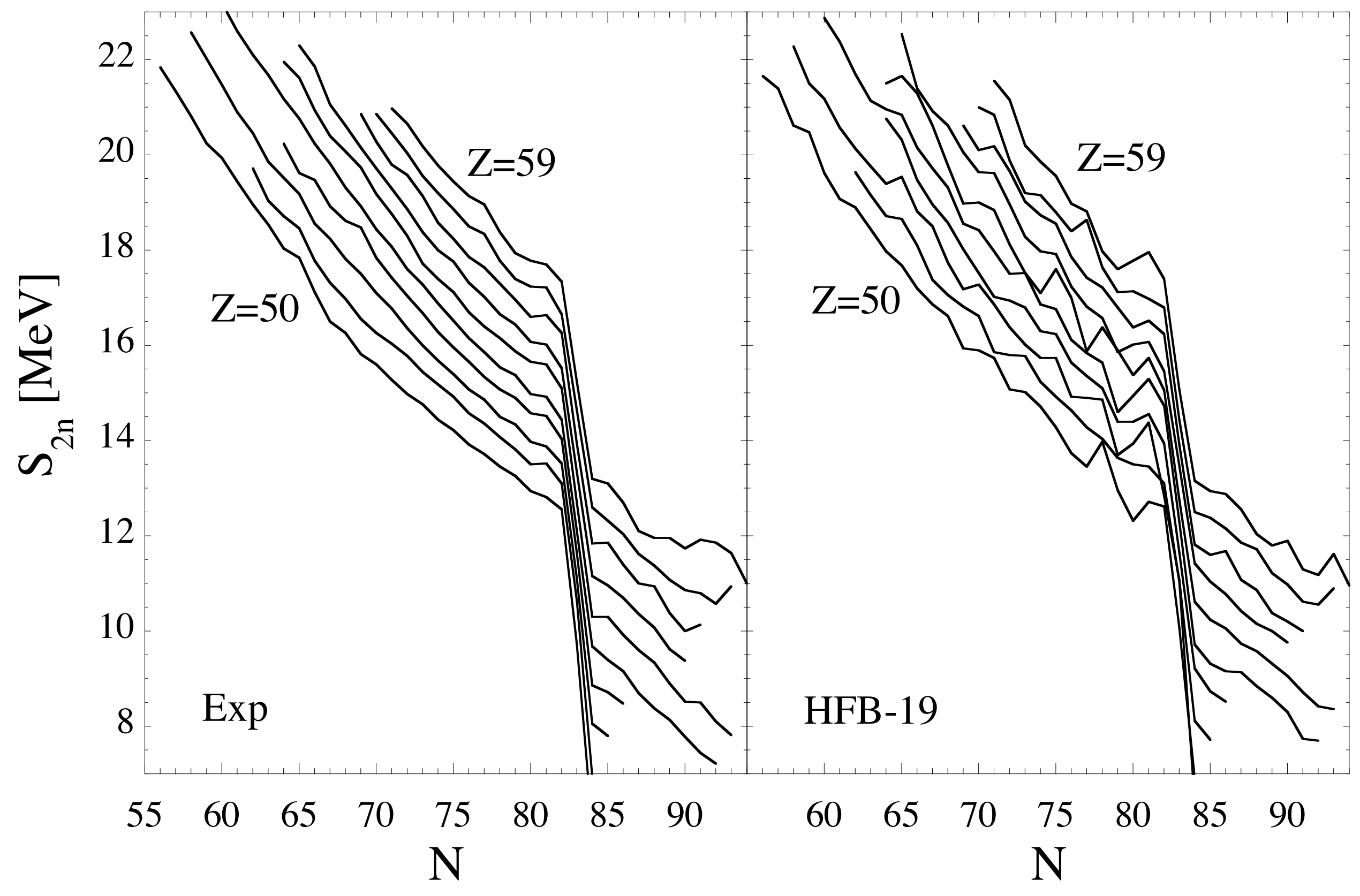




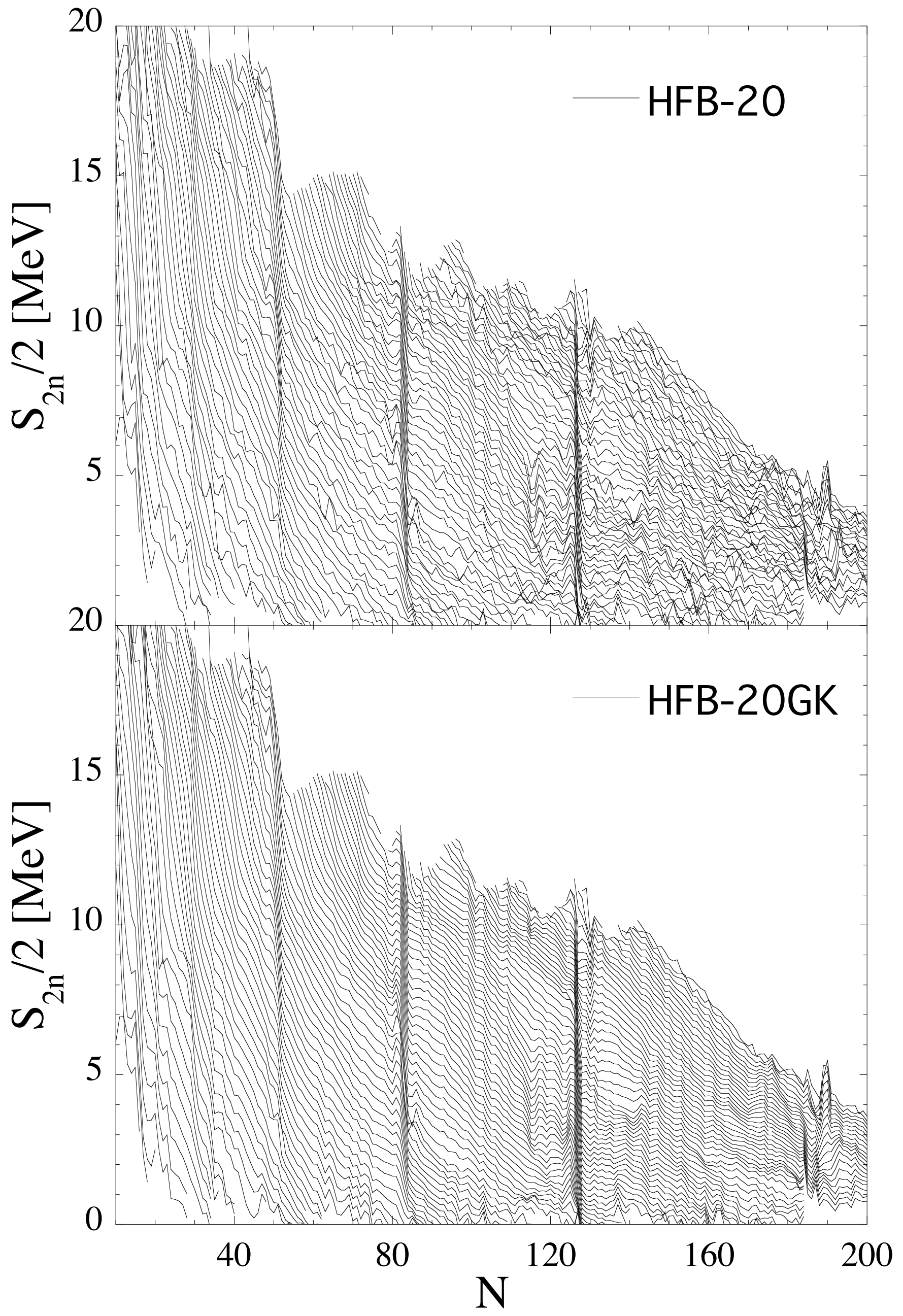




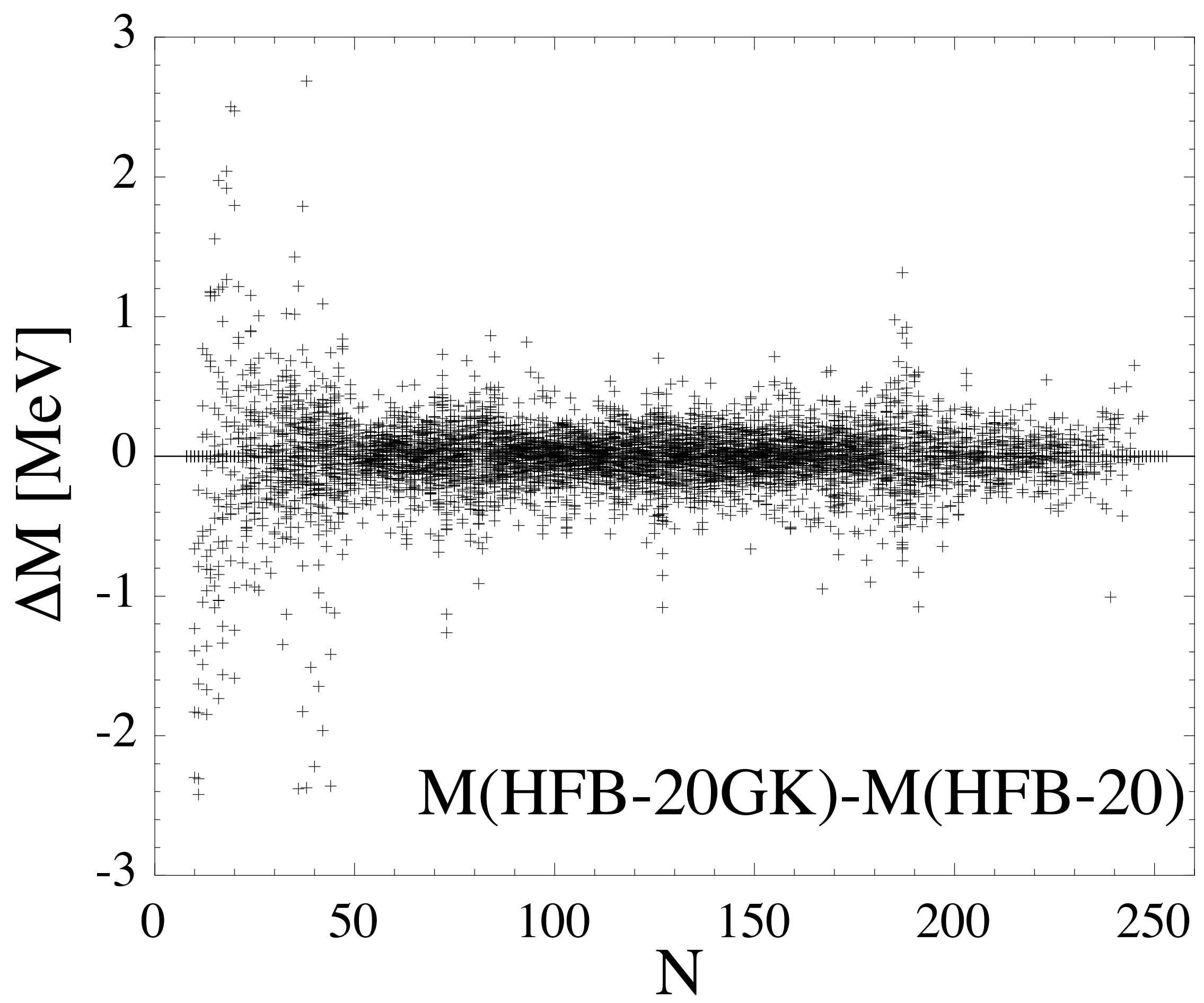

